\title{
REVISIÓN: MAQUINARIA USADA PARA LA ELABORACIÓN DE SURFACTANTES ANIÓNICOS DERIVADOS DEL ACEITE DE PALMA
}

\section{REVIEW: EQUIPMENT FOR ANIONIC SURFACTANT MANUFACTURE FROM OIL PALM}

\author{
Jesús Alfonso Torres Ortega ${ }^{I}$
}

RESUMEN

En el presente estudio, se realiza una exploración de los procesos actuales de sulfonación de diversas materias primas, para determinar las condiciones generales del proceso, con el objeto de presentar el estado del arte de los procesos de sulfonación para la fabricación de surfactantes anionicos. Se ha realizado una revisión bibliográfica haciendo énfasis en varios aspectos: Tecnologías de sulfonación, reactores y condiciones de operación en el proceso de sulfonación, técnicas analíticas para el seguimiento de la reacción, los modelos matemáticos propuestos en la literatura científica para la sulfonación/sulfatación en absorbedores tubulares, patentamiento y publicaciones de la industria especializada en el área de la sulfonación. Una revisión de los reactores de sulfonación utilizados comúnmente para la fabricación de tensioactivos derivados de compuestos orgánicos permite examinar las condiciones de diseño de los equipos así como las características integrales del proceso.

Palabras clave: anionico, reactor, sulfonación, tecnología, tensioactivo.

\begin{abstract}
In the present study is performed a review of the current processes of sulfonation of various raw materials for determine the process conditions in order to present the state of the art of sulfonation processes for the manufacture of anionic surfactants. There has been a scientific literature with emphasis on several aspects: Technology, sulfonation reactors and operating conditions in the process, analytical techniques for monitoring the reaction degree, the mathematical models proposed in the literature for the sulfonation/sulfation in tubular absorbers, patenting and specialized industry publications in this area.
\end{abstract}

Key word: anionic, reactor, sulfonation, surfactant, technology.

Aprobado: Junio 29 de 2010

1 Ingeniero Químico, MSc., PhD. Profesor Asociado del Programa de Ingeniería Ambiental y Sanitaria de la Universidad de La Salle, Bogotá, Colombia. jatorres@unisalle.edu.co. 


\section{INTRODUCCIÓN}

En el año 2008 la producción mundial de tensioactivos fue de 13 millones de toneladas alcanzando un volumen de ventas de EE.UU. de \$24,33 millones en 2009, lo que significó un incremento del $2 \%$ respecto al año anterior. Se prevé entonces un fuerte crecimiento de cercano al 2,8\% anual hasta 2012 y entre 3,5 - 4\% en los años venideros (Resnik et al, 2010). Por otra parte se debe tener en cuenta que las plantas industriales de sulfonación se encuentran dispersas en todo el mundo y estas cuentan con diferentes capacidades de producción que oscila entre 3,000 hasta 50,000 toneladas / año, principalmente para los tensioactivos anionicos. Por lo menos 800 plantas de sulfonación se estima que actualmente operan en todo el mundo. Sin embargo, alrededor del $20 \%$ de la producción mundial (2.500.000 toneladas / año de tensioactivos anionicos sulfonados) se concentra en los Estados Unidos, Europa Occidental y Japón (Inteligencia Acmite Mercado, 2010).

El tensioactivo es el componente clave en la formulación de detergentes. Una molécula de tensioactivos se compone de un lipofílicas "cola", generalmente una molécula orgánica C12-C14 (aceite soluble) y una "cabeza" soluble en agua (hidrofílico como el $\mathrm{SO}_{3}$ ). Mezclas de moléculas orgánicas, ya sea extraído de recursos no renovables, como el petróleo crudo, o de fuentes renovables como los derivados de aceites vegetales, son actualmente utilizadas como materias primas para la fabricación de detergentes domésticos.

El proceso de limpieza realizado por los tensioactivos anionicos (detergentes activos) se caracteriza por los siguientes eventos (de Groot, 1991):

(i) la humectación del sustrato y la suciedad debido a la reducción de la tensión superficial;

(ii) remoción de la suciedad del sustrato;

(iii) Mantenimiento de la suciedad en una solución estable o suspensión.

En este trabajo se presenta una revisión de la combinación de fenómenos que acontecen en la película descendente: Convección - difusión - reacción; éstos acompañan la hidrodinámica del modelo junto con la transferencia de materia y calor, aplicados a la absorción de SO3 sobre la mezcla de ésteres metílicos (Merchuk \& Farina, 1976; Mann \& Moyes, 1977; Nielsen \& Villadsen, 1983, 1985; Villadsen \& Nielsen, 1986; Bhattacharya et al. 1988).

\section{Sulfonación}

La sulfonación es el término que identifica a una reacción química electrofílica, en donde un grupo sulfónico - $\mathrm{SO} 3 \mathrm{H}$ se incorpora a una molécula con la capacidad de donar electrones. El producto de esta reacción química es reconocido como un ácido sulfónico, si la molécula donante de electrones es una de carbono. Anhídrido sulfúrico reacciona fácilmente con densidades electrónicas deslocalizadas como las presentes en los grupos aromáticos o alquenos. Estas reacciones producen una variedad de productos, incluyendo polisulfonas

derivados. Por otro lado, el proceso de sulfatación implica la incorporación de las moléculas -SO3H a un átomo de oxígeno en una molécula orgánica para formar enlaces C-O-S y el 
grupo de sulfato (Figura 1). Estos sulfatos ácidos pueden ser fácilmente hidrolizado, y por esta razón es necesaria una neutralización inmediata después de que el grupo de sulfato se ha formado (Foster, 1997). Aunque los procesos de sulfonación y sulfatación se emplean industrialmente para obtener una amplia gama de productos que van desde tintes para el cabello hasta pesticidas y productos intermedios orgánicos, sus principales aplicaciones están en la producción de tensioactivos anionicos (Foster, 2004). En la práctica se utilizan como material de base para los detergentes mezclados con moléculas orgánicas, ya sea a base de aceite mineral, de petróleo crudo o de productos renovables. Las materias primas orgánicas más importantes son: Lineal alquilbenceno, Alcohol primario, Éter alcohol primario, Alfaolefinas, Metil éster derivados de ácidos grasos.

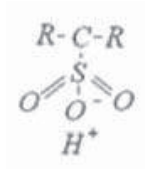

(a)

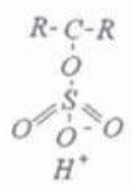

(b)

Figura 1. Grupos funcionales (a) sulfonato: $-\mathrm{SO} 3 \mathrm{H}$ y (b) sulfato: $-\mathrm{OSO} 3 \mathrm{H}$ (Behler et al., 2001)

\section{Avances en el siglo $\mathbf{X X}$}

Las primeras patentes sobre sulfonación de materias primas grasas aparecen hacia principios del siglo XX. Russ en 1913 (US Patent 1,081,775) recomienda un proceso de sulfonación por medio de la mezcla en un tanque agitado de aceite, ácido sulfúrico y una solución salina por espacio entre 24 y 48 horas. La separación del producto se realizaba por medio de lavados. Levinstein (US Patent 1,185,213) propone sulfonar ácido palmítico o palmitina con ácido sulfúrico y la ayuda de un aceite vegetal puro, en un tanque agitado refrigerado, con el fin de mantener la temperatura en $40^{\circ} \mathrm{C}$. El producto final es una dispersión de ácido palmítico sulfonado, con algunas aplicaciones surfactantes. La sulfonación con $\mathrm{SO}_{3}$ gaseoso ya aparece en patentes de 1919; Downs (US Patent 1,321,994) propone la sulfonación de hidrocarburos con $\mathrm{SO}_{3}$ gaseoso, en un proceso por lotes en un tanque agitado. Estas patentes se concentraban más en el procedimiento a seguir para obtener altos rendimientos de materia sulfonada que en el diseño de un equipo que permitiera obtener un producto dentro de las especificaciones requeridas. Las primeras sulfonaciones de materias grasas fueron procesos largos que se realizaban en tanques agitados sin ninguna exigencia especial de la chaqueta.

Para esta época existían dos grandes tendencias en sulfonación de materias primas grasas: Sulfonación de ácidos saturados en posición alfa y la sulfonación de ácidos insaturados o con grupos funcionales. Los problemas de color de esta última opción, llevaron a su progresivo abandono. Hasta ese momento el manejo de la viscosidad no se considera un factor importante. La sulfonación de materias petroquímicas era ya ampliamente conocida y medianamente desarrollada debido a la facilidad para sulfonar grupos aromáticos, en contraposición a la 
dificultad para sulfonar cadenas alifáticas. En 1933 Günther \& Hetzer (US Patent 1,926,442), siguen usando reactores agitados para la sulfonación de ácidos carboxílicos de cadena larga pero éstos comienzan a ser un tanto más sofisticados, por cuanto la temperatura de sulfonación propuesta es de aproximadamente $100^{\circ} \mathrm{C}$. Una agitación eficiente sigue considerándose como un factor importante. También se propone el uso de sustancias que diluyan el agente de sulfonación, práctica muy difundida en la mayoría de sulfonaciones por lotes, y cuyo fin es manejar la exotermicidad de la reacción (Gilbert, 1951). La sulfonación usando películas delgadas y agentes de sulfonación diluidos líquidos o gaseosos, ya había sido sugerida por Law (US Patent 2,088,027) en 1937 para la sulfatación de alcoholes grasos. El tipo de reactor que proponían era un tubo de vidrio de 0.25 pulgadas de diámetro y 45 pies de longitud, formando una espiral de 3 pulgadas de diámetro. $\mathrm{El} \mathrm{SO}_{3}$ al 3\% fluía a contracorriente diluido con nitrógeno. Se incluía un lazo de recirculación para llegar a conversiones adecuadas.

Las primeras iniciativas para sulfonar ésteres metílicos de ácidos grasos pueden vislumbrarse en la patente de Wendell \& Decatur en 1938 (US Patent 2,195,145). El agente de sulfonación propuesto es una mezcla de $\mathrm{SO}_{3} / \mathrm{SO}_{2}$, y el sistema de reacción no varía con respecto al propuesto anteriormente para la sulfonación de ácidos grasos saturados: un tanque agitado hermético, con un buen sistema de acondicionamiento de la temperatura y de agitación. La temperatura de sulfonación recomendada está alrededor de $40^{\circ} \mathrm{C}$. Syracuse (US Patent 2,195,187) en 1940, presenta la sulfonación de sales de ácidos grasos saturados disueltos en $\mathrm{SO}_{2}$, usando $\mathrm{SO}_{3}$ como agente sulfonante. Además de la buena agitación y buena temperatura, el uso de $\mathrm{SO}_{3}$ añade un requisito de hermeticidad para garantizar la seguridad en la reacción. El color se perfila como un parámetro significativo del producto final y empieza a relacionarse con la temperatura de reacción, razón por la cual se comienza a prestar más atención a los sistemas de calefacción.

Pueden encontrarse patentes sobre sulfonación de ésteres metílicos derivados de ácidos grasos, que datan de 1940 e incluso fechas anteriores para sulfonación de ácidos grasos. Wendell \& Decatur (US Patent 2,195,187) en 1938, hace reaccionar ésteres metílicos de aceite de coco hidrogenado mezclados con sebo, con $\mathrm{SO}_{3}$ líquido destilado de óleum, en presencia de $\mathrm{SO}_{2}$ líquido, bajo presión, $25^{\circ} \mathrm{C}$, condiciones anhidras, y por un período de 24 horas. La relación molar utilizada fue de 3 a 1 . El tipo de reactor usado fue un reactor batch. Moyer (U.S Patents 2,195,186; 2,195,187 y 2,195,188), en 1940, hace reaccionar ésteres metílicos de aceite de coco hidrogenado mezclados con sebo, con $\mathrm{SO}_{3}$ líquido destilado de óleum, en presencia de $\mathrm{SO}_{2}$ líquido, bajo presión, $25^{\circ} \mathrm{C}$, condiciones anhídridas y por un período de 24 horas. La relación molar utilizada fue de 3 a 1 . El tipo de reactor usado fue un reactor batch. Eaton et al. en 1944 (US Patent 2,352,698) proponen sulfonar una mezcla de un ácido graso y un alcohol monohídrico, empleando ácido sulfúrico y un control minucioso de temperatura en dos etapas, propuesta que requiere sistemas de calentamiento y refrigeración muy efectivos. Una primera etapa entre 60 y $100^{\circ} \mathrm{C}$ y una segunda entre 35 y $0^{\circ} \mathrm{C}$. 
Los sistemas de sulfonación continua con $\mathrm{SO}_{3}$ en película fina se popularizan en la década de los 50, y se usan extensivamente para materias primas petroquímicas. La tecnología de reacción en película fina y el manejo de $\mathrm{SO}_{3}$ se extiende a materias primas oleoquímicas rápidamente. El solvente como el dimetilsulfóxido, o el tetrahidrofurano, que en las sulfonaciones por lotes se usaba para diluir el agente de sulfonación, es sustituido por aire seco o el nitrógeno deshumidificado. Las patentes se concentran en los mecanismos de generación de $\mathrm{SO}_{3}$ y en diseñar novedosos sistemas de contacto.

A finales de los 50 y principios de los 60, la tecnología de reactores de película se consolida. Falk et al. en 1960 (US Patent 2,923,728) propone un reactor tubular horizontal, en el cual la materia orgánica es impulsada por medio de un cabezal a gran velocidad, de tal manera que se logra un flujo anular. En el interior del anulo, se hace fluir $\mathrm{SO}_{3}$ entre el 12 y el $22 \%$, diluido con un gas inerte a una velocidad tal que garantice el flujo anular de la carga orgánica. La velocidad del gas estaba entre 45 y $122 \mathrm{~m} / \mathrm{s}$. El flujo de $\mathrm{SO}_{3}$ era del orden de 0.3 $\mathrm{kg} / \mathrm{hr}$. El reactor fue probado para sulfatar alcoholes grasos, obteniendo una materia activa de $35 \%$.

La relación entre agente sulfonante y materia orgánica grasa comienza a ser muy tenida en cuenta en las patentes de los años 50 con lo cual las características del reactor moderno de sulfonación se van definiendo poco a poco (Gilbert, 1951). Rueggeberg \& Sauls en 1953 (US Patent 2,743,288), recomiendan una relación de 1 a 1.25 moles de $\mathrm{SO}_{3}$ por mol de materia grasa. En su patente, también sugieren el uso de un sulfonador continuo que hace uso de un cabezal en forma de disco rotatorio (spinning disk sulfonator), el cual permite la formación de películas delgadas compuestas por una mezcla de la materia a sulfonar (ácidos grasos insaturados) y $\mathrm{SO}_{3} / \mathrm{SO}_{2}$ líquidos. El principal problema de las sulfonación es batch de materias grasas, radicaba en la adecuada eliminación de agua y en los métodos para separar el agente sulfonante remanente, del producto final.

\section{Etapas del proceso de sulfonación}

La reacción de sulfonación es altamente exotérmica y la viscosidad del ácido orgánico producido (500-1000 centi Poise) es significativamente mayor que la viscosidad de los insumos (5-10 centi Poise). En el caso del lineal alquilbenceno se requiere una fase de hidrólisis o de estabilización necesaria para convertir el anhídrido formado en lineal alquilbenceno sulfónico. El alcohol y los ácidos sulfónicos etoxilato de alcohol, así como los metil esteres sulfonados requieren de una etapa de digestión (reorganización o envejecimiento) para alcanzar una alta conversión en el producto, además debe ser neutralizada inmediatamente después de la digestión para evitar la formación de subproductos intermedios indeseables (Figura 2). Después del envejecimiento y la hidrólisis se obtiene un producto estable puede ser almacenado y transportado. 


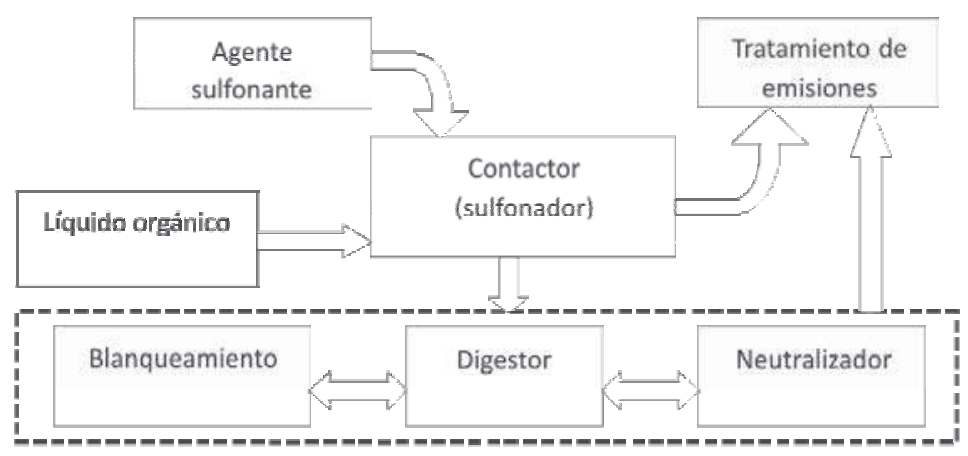

Figura 2. Diagrama del proceso industrial de sulfonación (MacArthur et al., 1998)

Las reacciones de neutralización pueden llevarse a cabo con muchos productos químicos como el hidróxido de sodio. La neutralización con sosa cáustica diluida puede ser caracterizada como una reacción altamente exotérmica e instantánea. Diferentes tipos de reactores en cascada (Figura 3), que consisten de una bomba de circulación, homogeneizadores (donde el ácido se introduce en solución alcalina que circulan) e intercambiadores de calor, se usan para la etapa de neutralización.

Las siguientes son consideraciones a tener en cuenta para los procesos de sulfonación:

(i) Los agentes sulfonantes son productos químicos altamente corrosivos y peligrosos como el oleum o ácido sulfúrico concentrado con trióxido de azufre pueden formar neblinas ácidas y sus procesos producen temperaturas elevadas;

(ii) Las reacciones de sulfonación y neutralización son muy delicados en el sentido de que estas operaciones conducen a la generación de gran cantidad de subproductos indeseables que colorean intensamente el producto y ocasionan un bajo rendimiento de materias primas orgánicas.

(iii) las operaciones requieren un control adecuado que prevenga situaciones de peligro para los operarios y las instalaciones. No tener en cuenta las normas de funcionamiento seguro no sólo tendrá como resultado productos de baja calidad y bajos rendimientos, sino que también reducirá seriamente la vida útil de la planta.

\section{Aparatos empleados para la sulfonación de grasas/aceites}

Blazer et al. en 1964 (US Patent 3,158,632) proponen el sistema de reacción que se muestra en la figura 3(a) para la sulfonación continua de ácidos grasos y sus derivados. Se inicia la sulfonación a $40^{\circ} \mathrm{C}$ y en el intercambiador alcanza los $70^{\circ} \mathrm{C}$. Se obtienen aproximadamente 
$171 \mathrm{~g} / \mathrm{h}$ de éster sulfonado. En esta misma patente, se propone un equipo de reacción alterno para sulfonar con $\mathrm{SO}_{3}$ gaseoso, constituido por 4 reactores batch conectados en cascada (operando a $50,60,70$ y $85^{\circ} \mathrm{C}$ ) y un reactor de terminación, manejando un gradiente de temperaturas en cada uno de ellos figura 3(b). El conjunto de reactores CSTR en cascada opera en forma continua se utiliza para la separación del producto de manera similar al reactor sulfurex que actualmente comercializa Ballestra. La ventaja de este sistema consiste en la posibilidad de manejar el flujo de $\mathrm{SO}_{3}$ que entra a cada reactor, y de esta manera tener un mejor control de la reacción y del color del producto final.

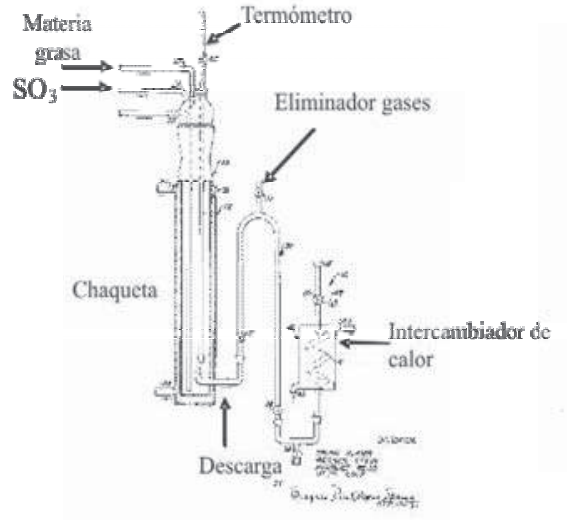

(a)

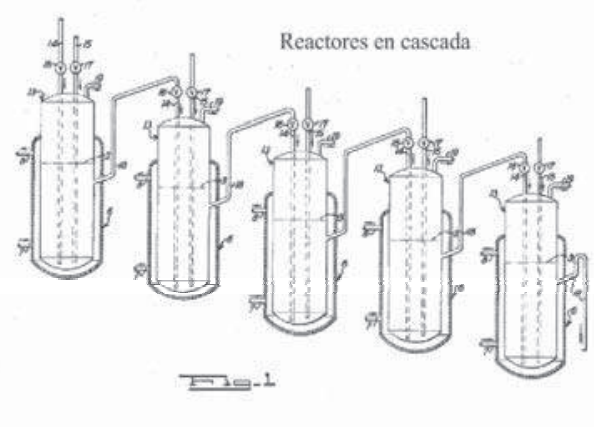

(b)

Figura 3. (a) Dispositivo de absorción para la sulfonación de ácidos grasos; (b) Sistema de sulfonación en cascada (Blazer et al., 1964)

Stepan Company en 1965, por intermedio de Knaggs (US Patents 3,169,142), proponen un reactor de película multitubular propiamente dicho, para la sulfonación de gran variedad de materias primas, entre ellas ésteres metílicos de ácidos grasos. Stirton et al. (1965) reportan la sulfonación batch de ácidos grasos desde láurico, hasta bencénico, adicionando trióxido de azufre líquido, al ácido graso disuelto o disperso en cloroformo. La relación molar entre ácido y $\mathrm{SO}_{3}$ varió entre 1.5 y 1.7 , la temperatura de reacción alcanzó los $65^{\circ} \mathrm{C}$. El tiempo de reacción fue de 1 hora, alcanzándose rendimientos ente 75 y 85\%. El color no obstante no fue satisfactorio.

El reactor de película descendente construido por Hulbert (1967) para la sulfonación de éster metílico de sebo hidrogenado en el Witco Technical Center, vislumbra los elementos de los sistemas de reacción actuales (Figura 4). 
Revista Especializada en Ingeniería de Procesos en Alimentos y Biomateriales. Unad.

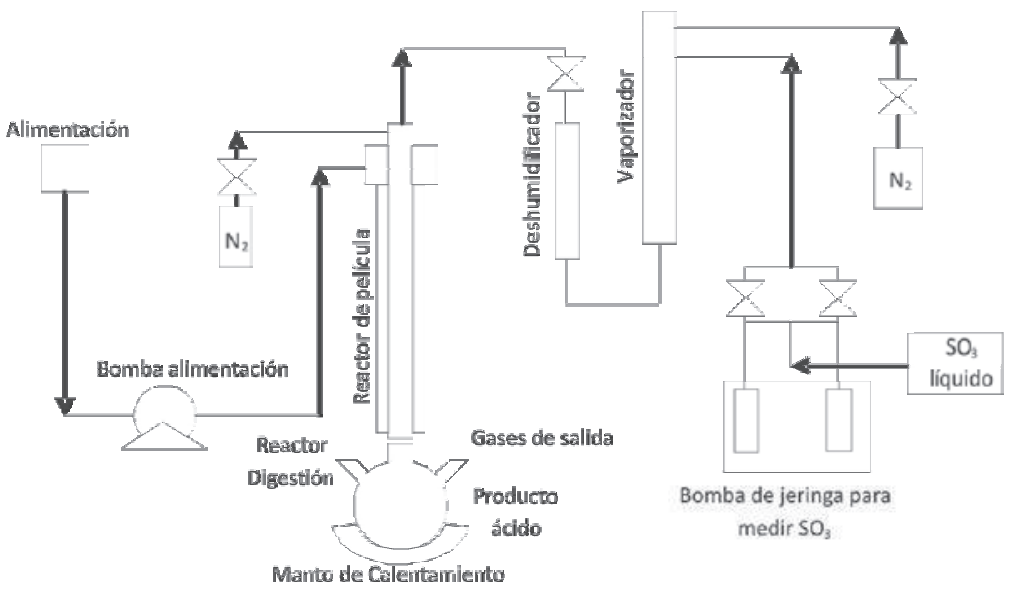

Figura 4. Aparato utilizado en la experimentación realizada por Hulbert (1967)

Stein \& Baumann (1975), utilizan un esquema de sulfonación mostrado en la figura 5 en el que mediante un banco de reactores anulares de película Chemithon sulfonan metil estearato de palma entre otros compuestos, obteniendo muy buenos resultados cercanos al 50\% de éster sulfonado y neutralizado. Ellos profundizan el estudio para las etapas de blanqueamiento y neutralización considerándolas como los pasos críticos del proceso. Finalmente evalúan las propiedades como detergente de los MES obtenidos en distintas soluciones.

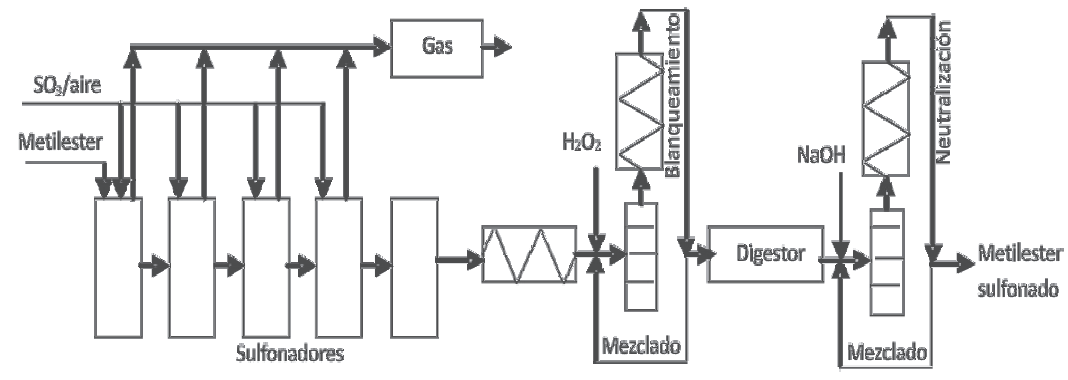

Figura 5. Esquema de la fabricación de esteres grasos sulfonados (Stein \& Baumann, 1975)

Gutiérrez et al. (1988), construyen un sistema para la sulfonación con $\mathrm{SO}_{3}$ líquido estabilizado y que posteriormente Talens (1999) también utilizaría, proponen el sistema de reacción mostrado en la figura 6 para la sulfonación de alquilbencenos lineales. 


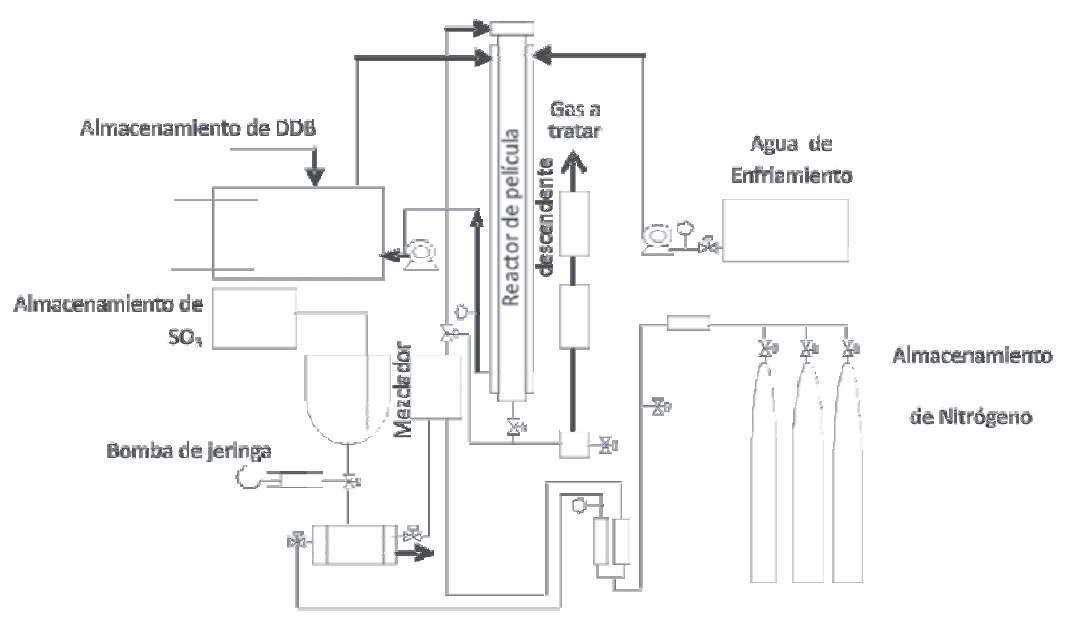

Figura 6. Esquema del sistema utilizado en la experimentación realizada por Gutiérrez et al. (1988)

En la tabla 1 se aprecia las principales condiciones de operación para los sistemas utilizados entre los años 60 y 80 para la sulfonación de diversas materias primas. Chemithon Corporation implemento un sistema de sulfonación desarrollando un reactor de película de tubos concéntricos de $2 \mathrm{~m}$ de longitud con un flujo anular de líquido orgánico, consistente de dos partes, la primera un flujo pistón de mezcla perfecta y la segunda por una serie de tubos, es esencial la remoción de calor en este sistema, como se observa en la figura 7. Hovda (1993) trabaja con el sistema de reacción que se muestra en la figura 8 para sulfonar ésteres metílicos, incluye las etapas de neutralización y blanqueo. Como gas de arrastre se usa aire seco, con un punto de rocío de $-55^{\circ} \mathrm{C}$. El trióxido de azufre, se prepara en planta, por oxidación catalítica del $\mathrm{SO}_{2}$. El éster metílico usado tiene un índice de Yodo menor de 0.5. Para la reacción se utiliza un reactor anular Chemithon, con recirculación de ácido sulfónico en una relación de 10 a 1 . Antes de entrar al reactor, la corriente de reciclo es refrigerada. Utiliza un ciclón como equipo especializado para separar las fases. La etapa de digestión se lleva a cabo de manera continua, en un digestor de flujo pistón bafleado. La temperatura de digestión está entre 80 y $95^{\circ} \mathrm{C}$, y el tiempo de residencia entre 16 y 30 minutos. El blanqueo se realiza en una malla de blanqueo, en un equipo que no sea metálico (se evidenció que el uso de equipos metálicos influye fuertemente en el color). La neutralización es realizada en un "loop reactor" con hidróxido de sodio acuoso. 
Revista Especializada en Ingeniería de Procesos en Alimentos y Biomateriales. Unad.

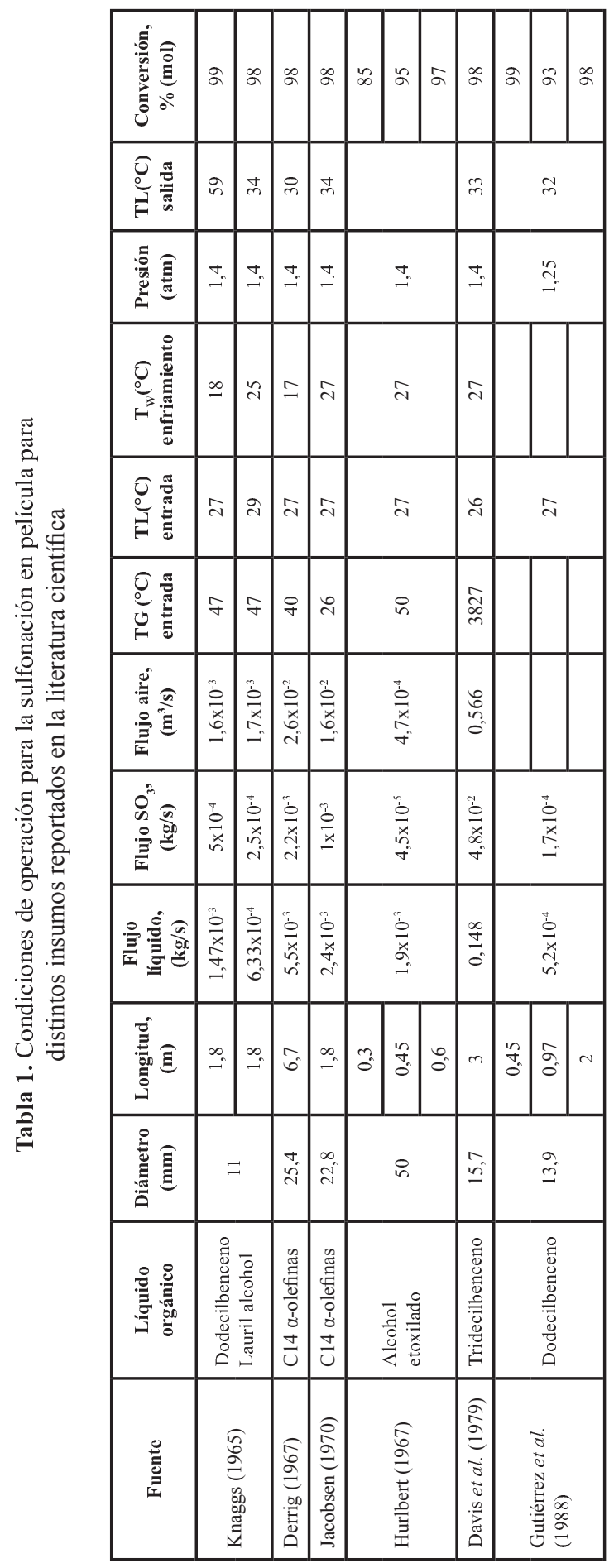




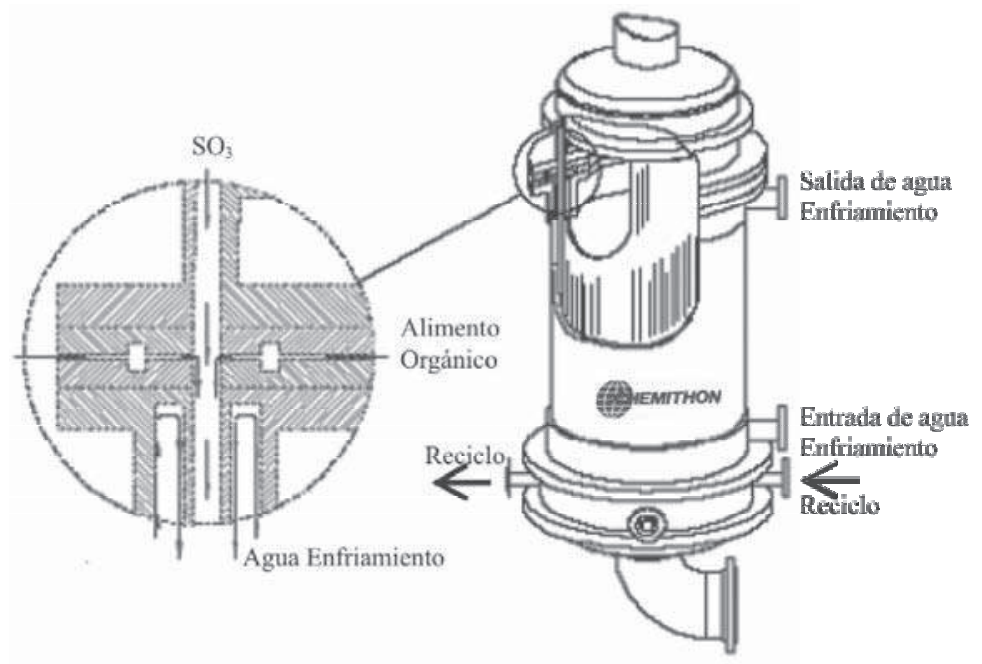

Figura 7. Detalle de las boquillas del reactor de tubos concéntricos FFR (de Groot, 1991)

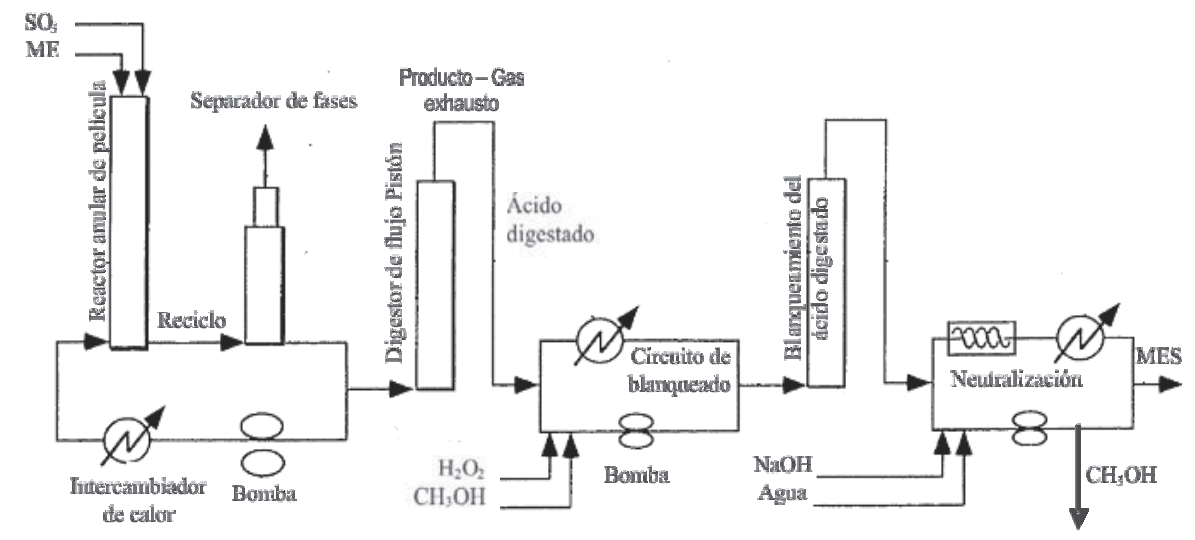

Figura 8. Esquema usado en la optimización realizada por Hovda (1993)

Talens (1999) utiliza el esquema mostrado en la figura 9 utilizando un FFR liso para sulfatar alcoholes etoxilados con $\mathrm{SO}_{3}$ vaporizado, su trabajo mostro un avance en modelamiento al determinar una transición entre las velocidades del líquido orgánico sulfonado y sin sulfonar. Por su parte Ahmed et al. (2000), uso un reactor de película pero con ondulaciones para la sulfonación de dodecilbenceno con SO3 que se observa en la figura 10, y los comparo frente a los datos de Gutiérrez et al. (1988), encontrando un mayor rendimiento en el contenido de la materia activa producida. 
Revista Especializada en Ingeniería de Procesos en Alimentos y Biomateriales. Unad.

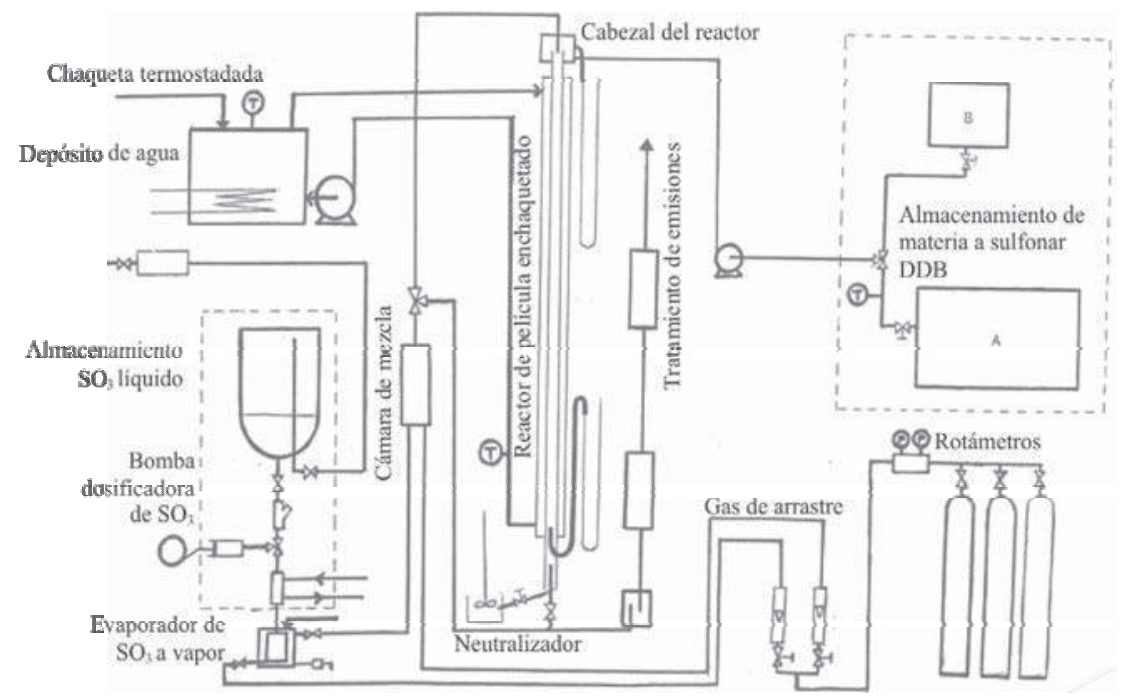

Figura 9. Sistema de reacción para sulfonación de DDB con SO3 empleado por Talens (1999)

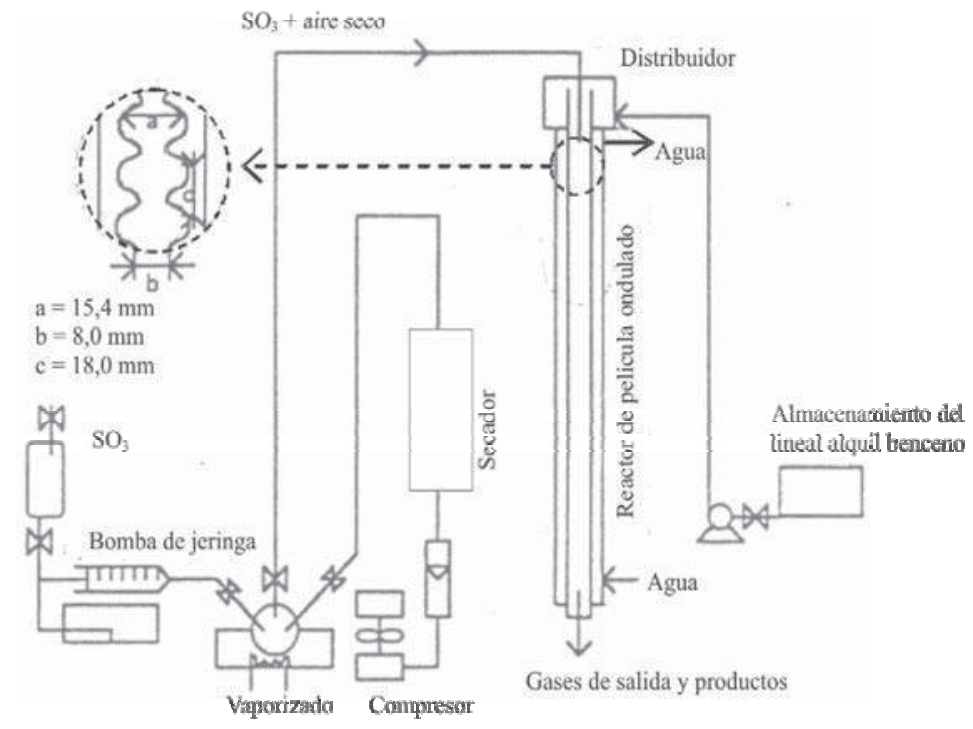

Figura 10. Sistema de experimentación usado por Ahmed et al. (2000) a través de un FFR ondulado

\section{Estudios de adsorción para reacciones exotérmicas}

A nivel de laboratorio, esta concentración sería deseable, pero comúnmente sólo se pueden conseguir concentraciones de hasta $25 \%$ grado técnico. Merck comercializa óleum a 
concentraciones más altas, hasta $65 \%$ con grado reactivo. Autores de gran trayectoria en el campo de la investigación en sulfonación de metil ésteres como Ahmad et al. (1998) usan óleum al 20\% como medio para generar $\mathrm{SO}_{3}$. Ahmad trabajó en un reactor de película donado por Henkel, a $600 \mathrm{~g} / \mathrm{h}$. Como materias primas usó estearina de palma, la cual esterifico y la hidrogenó en laboratorio. La hidrogenación fue realizada en un reactor agitado, a $195^{\circ} \mathrm{C}, 5.5$ bar, y $500 \mathrm{rpm}$. Para genera el $\mathrm{SO}_{3}$ usó un vaporizador trabajando a $150^{\circ} \mathrm{C}$, el $\mathrm{SO}_{3}$ obtenido por esta vía, es de menor calidad que el que se obtendría con óleum al $60 \%$ o con el SO3 líquido estabilizado, razón por la cual los grados de sulfonación esperados van a ser bajos. A pesar de esto, el uso de óleum puede convertirse en una alternativa perfectamente válida.

Se hallan en la literatura otros estudios sobre sulfonación de esteres metílicos adelantados por variados autores para la etapa de digestión y blanqueamiento del producto sulfonado: (Fabry \& Giesen, 1990; de Groot, 1991; Yamada, 1996; Roberts, 1988, 1995, 2008; US Patents $3,169,142 ; 4,080,372 ; 5,587,500$ ); lo mismo sucede con la etapa de neutralización: (Stirton, 1962; Stein et al.,1970; de Groot, 1991; Foster, 2004; US Patents 5,391,783; 5,391,786; $6,657,071)$; sin embargo se carece de una profundización en la primera etapa del proceso que ocurre en el sulfonador. Por esto, se considera de especial interés adelantar un estudio intensivo sobre lo acontecido en esta primera etapa del proceso en el reactor de película descendente considerándola como una etapa primordial del proceso integral.

Varios autores analizan las variables significativas del proceso que determina el grado de sulfonación y la coloración del producto y efectuaron ensayos a nivel laboratorio (Gilbert et al., 1953; Kircher et al. 1954; Hulbert et al., 1967). En la literatura científica se encuentran otros estudios para la absorción con reacción exotérmica en columnas de película descendente (Mann \& Moyes, 1977; Nielsen \& Villadsen, 1983), además de estudios sobre modelamiento aplicados en reactores de película para la sulfonación de derivados petroquímicos, particularmente para el dodecilbenceno (Johnson \& Crynes, 1974; Davis et al., 1979; Gutiérrez et al. 1988; de Groot, 1991; Dabir, et al., 1996; Talens, 1999; Akanksha, 2007); sin embargo, ninguno realizado para ésteres metílicos, objeto de particular interés del presente estudio. La descripción fenomenológica de la transferencia de masa, la cantidad de movimiento y el calor, acontecidas en el reactor de película descendente bifásico para la reacción de sulfonación, permite el desarrollo de un modelo matemático que relaciona las variables geométricas y de proceso en el reactor, con la conversión, el perfil longitudinal de temperatura y la posición del plano de reacción (Torres et al. 2009).

\section{Reactor de película líquida descendente}

Un reactor de película descendente (FFR, por su acrónimo en inglés: Falling Film Reactor) es un contactor semejante a una torre de paredes mojadas, como se muestra en la figura 11, enchaquetada para que circule un fluido refrigerante, generalmente agua. 


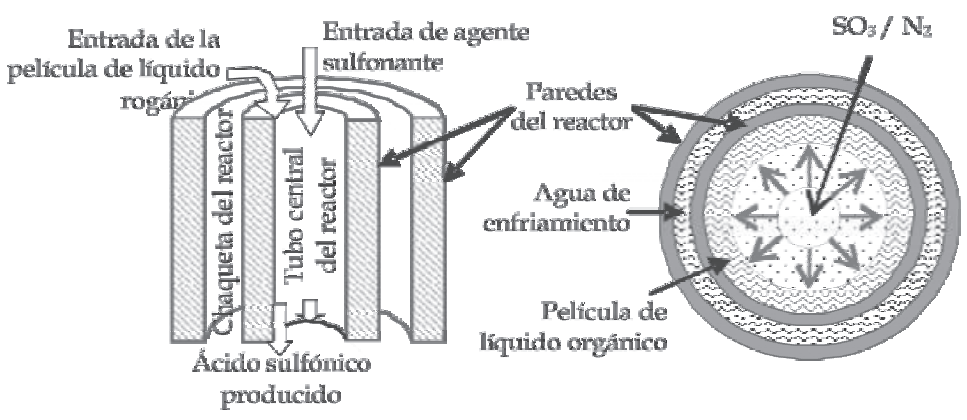

Figura 11. Esquema de un reactor de película (vista frontal y superior)

Estos dispositivos se han utilizado para estudios teóricos de transferencia de masa debido a que la superficie interfacial entre las fases mantiene un contacto directo. El flujo de alimentación debe ser medido y controlado con la mayor exactitud posible, el control del flujo de alimentación se realiza a través de una bomba de velocidad variable. Existen múltiples diseños para los reactores de película descendente, los cuales se diferencian en el esquema de refrigeración, la dirección de los flujos, en los tipos de boquillas empleadas para crear la película de la materia a sulfonar, y en la presencia y configuración de reciclos. Ballestra y Chemithon son dos empresas líderes en este tipo de tecnologías. Precisamente en el país la empresa Química Básica Colombiana posee un reactor industrial de película multitubo (FFR-MT) adquirido de Demex Ballestra SpA como el que se observa en posición horizontal en la figura 12. La caída de presión del gas en estas torres es probablemente menor que en cualquier otro aparato de contacto gas-líquido, para un conjunto dado de condiciones de operación.
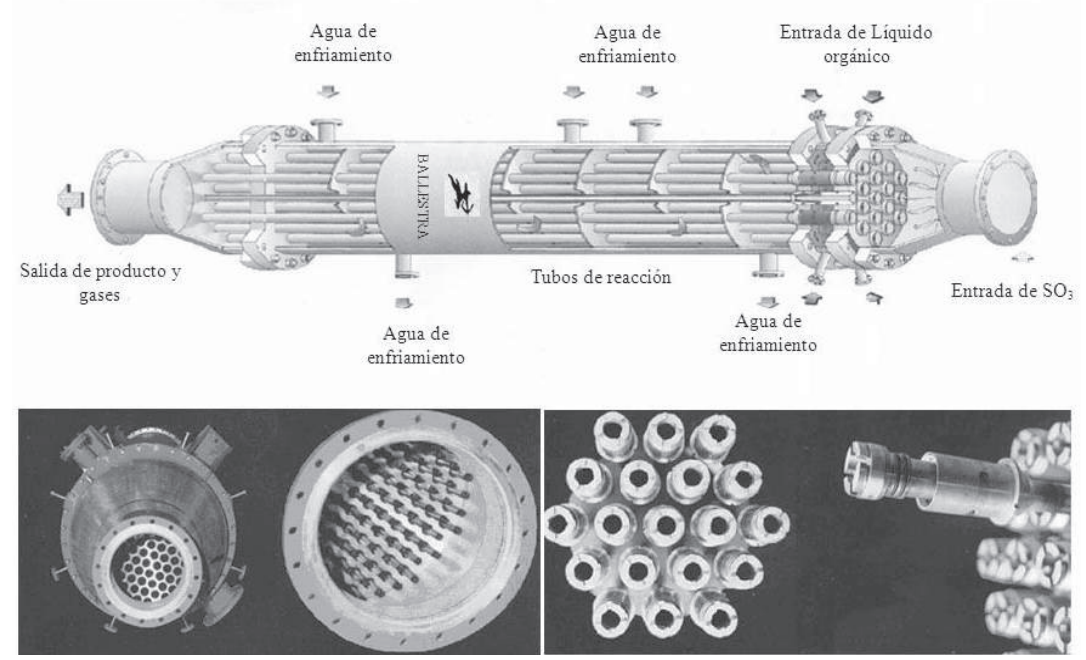

Figura12. Reactor de película multitubular (FFR-MT) R\&D Ballestra SpA (Moretti \& Adami, 2002) y detalles del cono superior, las boquillas en el cabezal de entrada y la base del FFR-MT de R\&D Ballestra SpA (Moretti \& Adami, 2002) 
Hay que tener en cuenta que si bien el FFR cuenta con las características ideales para reacciones de sulfonación, no es el único proceso en el cual se puede utilizar. Procesos de cloración y de etoxilación usan muy a menudo este tipo de tecnología. Dentro de los elementos claves de los diseños modernos de FFR se encuentra el cono superior o la cabeza del reactor en la cual se regulan los flujos de entrada al reactor y cuyo ajuste de los tubos debe ser mecánicamente hermético y preciso para garantizar la formación de una película uniforme de mínimo espesor con flujo laminar. De igual forma el ajuste de los tubos debe hacerse mecánicamente exacto en el plato inferior y permitir fácilmente la separación de los gases de salida.

Detalle de las boquillas de distribución patentadas por Ballestra SpA se observan en la figura 12; éstas permiten teóricamente operar un flujo de $10.000 \mathrm{Kg} / \mathrm{h}$ con precisión de \pm 0.005 . El número de tubos de un reactor de película es por lo general mayor a 100 aunque, en general, esto depende del flujo que se esté manejando. A nivel industrial la longitud de los tubos es cercana a los 5 y 7 metros y diámetros cercanos a los $20 \mathrm{~cm}$.

De las partes más importantes de un reactor de película y posiblemente la que mejor debe estar diseñada es el mecanismo de formación de la película. Esto es tan cierto, que para muchos fabricantes el esfuerzo de diseño del reactor de película se concentra en el diseño del cabezal. Un ejemplo de diseño de cabezales para la formación de película está consignado por Lanteri (1978) en la US Patent 3,931,273, una de las muchas patentes existentes sobre diseño de reactores de película que concentra su esfuerzo en el diseño de cabezales (Figura 13). Los cabezales donde se forma la película deben asegurar que ésta sea suficientemente delgada, se encuentre bien distribuida alrededor de todo el perímetro del tubo y que fluya de manera continúa.

Del aseguramiento de las características deseables de la película depende en gran medida el adecuado comportamiento térmico del reactor y la posibilidad que la relación agente sulfonante/ materia orgánica se mantenga constante a lo largo de todo el reactor. Así como la relación global entre el agente sulfonante/materia orgánica se controla mediante los flujos de materias primas, la relación local (en cada punto del reactor) se controla con un adecuado flujo de película.

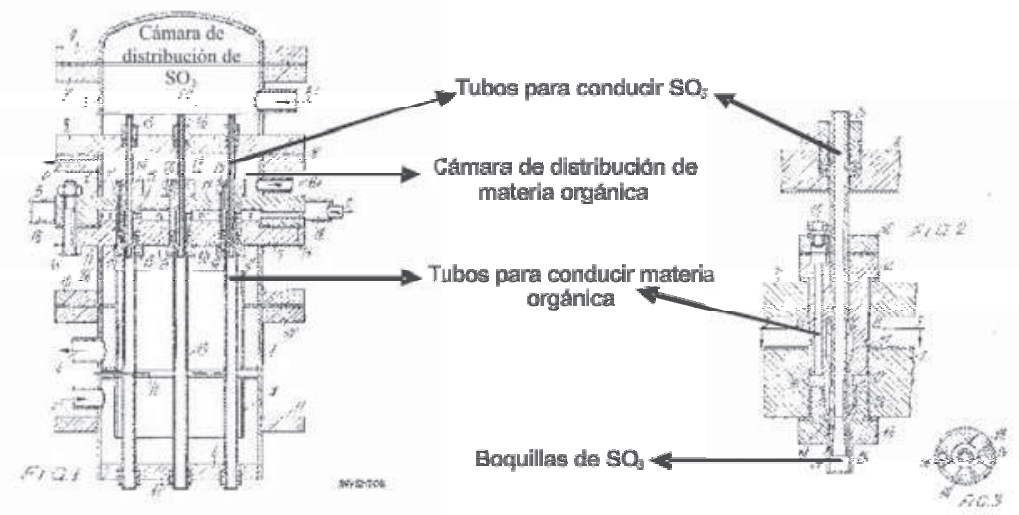

Figura 13. Cabezal de un reactor de película (Lanteri, 1978) 
En la figura 13 se pueden distinguir claramente una cámara superior por la cual entra el $\mathrm{SO}_{3}$ y se distribuye a través de las boquillas de un banco de tubos. Una segunda cámara, aislada de la primera y a través de la cual pasan los tubos que llevan el $\mathrm{SO}_{3}$, se encarga de recibir la materia orgánica y distribuirla en las boquillas que la llevan a los delgados canales que finalmente van a formar la película. El espesor de estos canales o coronas depende del fluido que va a alimentar al reactor, pero por lo general varía entre 0.25 y $0.35 \mathrm{~mm}$. Los empaques y sellos que ajustan los tubos a cada placa deben estar especialmente diseñados para evitar derrames o contacto de las materias primas antes de la zona de reacción.

Consideraciones para el diseño de equipos para sulfonación

Un reactor de película descendente para la sulfonación debe diseñarse para poder realizar un control minucioso sobre todas las características de la reacción. La tecnología de la sulfonación está muy bien desarrollada, madura y con un significativo nivel de especialización en el tratamiento de los derivados petroquímicos. Al cambiar la materia prima, aunque compartan muchas características propias del proceso, hasta cierto punto, las características de reacción cambian, de tal manera que una variable que se consideraba de suma importancia en el proceso convencional, puede tener otro comportamiento en el proceso, como la forma de interacción química de las materias primas o la termodinámica.

Debido a la gran rapidez de la reacción, que alcanza altos porcentajes de conversión en los primeros centímetros del reactor, al interior de éste se presenta una variación de la temperatura. Por ello, cuando se generaliza hablando sobre temperatura de operación en el reactor, debe hablarse de varias temperaturas a saber: La temperatura en el punto caliente que da una idea de cómo se está comportando la reacción y que puede estar entre los 65 y $75^{\circ} \mathrm{C}$. Es difícil controlar la temperatura del punto caliente ya que éste se desarrolla rápidamente y su duración está restringida a un pequeño tramo de longitud. Manejando las variables de proceso, lo único que puede hacerse es subir o bajar su localización.

La temperatura de salida del reactor y la temperatura de la chaqueta son mucho más fáciles de controlar y de medir por el manejo de las variables de operación. Medidas al mismo tiempo la temperatura de la chaqueta en varios puntos y la temperatura de salida compensan empíricamente la inexactitud que se puede tener con la medición del punto caliente. Temperaturas altas favorecen un descenso en la viscosidad y por lo tanto un flujo más rápido y un mayor contacto entre materia orgánica y el $\mathrm{SO}_{3}$, sin embargo pueden favorecer reacciones secundarias. Por el contrario, bajas temperaturas reducen las reacciones laterales, pero favorecen un aumento de viscosidad y una menor conversión (Figura 14). 
Revista Publicaciones e InVestigación • ISNN: 1900-6608 • Volumen 4 • Agosto de 2010
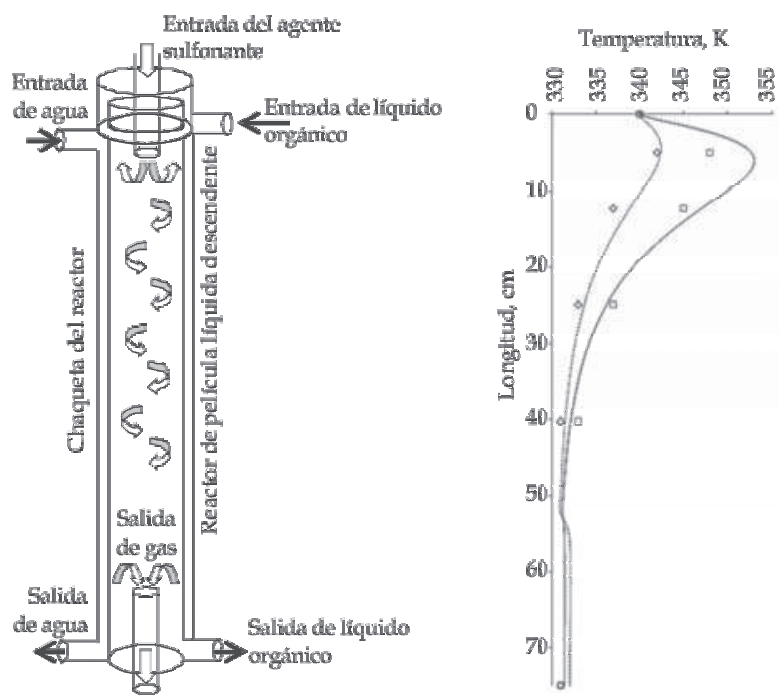

Figura 14. Representación de los cambios en la temperatura en la sulfonación dentro de un reactor de película (Torres, 2009)

Las investigaciones hablan de una relación directa entre la conversión (determinada por el grado de sulfonación), la temperatura y la coloración del producto sulfonado (US Patent No. 5,391,786). A mayores conversiones, se hace más difícil manejar el color del producto (U.S. Patent No. 4,671,900). El producto final de reacción: $\alpha$-sulfo metil estearatos derivados del aceite de palma, objeto de estudio, será por tanto un producto coloreado en mayor o menor grado de acuerdo con las condiciones de reacción (Yamada \& Matsutani, 1993). Las principales condiciones de diseño a tener en cuenta en una planta de sulfonación se presentan a continuación.

Generación del agente sulfonante, existen varias alternativas para la generación del agente de sulfonación, como el uso de trióxido de azufre líquido, el acople de la planta de sulfonación a una planta de trióxido de azufre, o el arrastre de trióxido de azufre de óleum (Castañeda \& Rivas, 2004). Industrialmente las plantas de sulfonación poseen un sistema propio de generación de $\mathrm{SO}_{3}$, lo que es muy recomendable para que permita obtener corrientes de $\mathrm{SO}_{3}$ a las concentraciones requeridas; tal es el caso de la empresa Química Básica Colombiana que cuenta con una planta contigua de producción de ácido sulfúrico. A grandes rasgos, la generación de $\mathrm{SO}_{3}$ empieza con la fusión de azufre, la oxidación del azufre fundido con corrientes de oxígeno para formar $\mathrm{SO}_{2}$ gaseoso, seguida de la posterior oxidación catalítica usando pentóxido de divanadio $\left(\mathrm{V}_{2} \mathrm{O} 5\right)$ como catalizador en un reactor empacado para finalmente formar el $\mathrm{SO}_{3}$ gaseoso. $\mathrm{El} \mathrm{SO}_{3}$ líquido tiene el inconveniente con los costos de inversión y de los riesgos que representa su adecuado manejo. La alta reactividad de la 
mayoría de agentes sulfonantes (especialmente del $\mathrm{SO}_{3}$ ), junto con la alta exotermicidad de la reacción, generan una serie de productos indeseables ya sea por su coloración intensa o porque afectan el desempeño del surfactante (Roberts, 1995).

Presión de operación, la reacción de sulfonación procede espontáneamente a un poco de presión superior a la atmosférica, razón por la cual no hay necesidad de trabajar con presiones altas. La presión de entrada debe ser mayor a la atmosférica para generar caída de presión, pero no hay un requisito de presión de entrada o presión en el reactor para llevar a cabo la reacción más que por las condiciones hidrodinámicas de los flujos utilizados en el proceso. El comportamiento del reactor de película se puede considerar desde cierto punto de vista como un absorbedor no isotérmico (Díaz, 2009). Como en toda operación de absorción un aumento en la presión favorecería la transferencia de masa; en el caso de la reacción sulfonación, por la alta velocidad de reacción y la alta exotermicidad que la caracteriza, es necesario de alguna manera controlar la transferencia de masa dentro del reactor para controlar así la velocidad de reacción. Por lo tanto no es necesario un sistema de presurización. La fase gaseosa va a entrar a una cierta presión (no muy alta) y, rigurosamente hablando, dependiendo del gas de arrastre, en el reactor se va a presentar un perfil de presiones debido a la caída de presión del gas que asegure que el gas desarrolle velocidades adecuadas para no afectar el flujo anular de la película. La presión de salida va a ser un tanto mayor a la atmosférica debido a la presión del flujo de nitrógeno. En un reactor Ballestra, la caída de presión puede ser de 4 psig. En un reactor Mazzoni, la caída de presión en los tubos es del orden de 3 psig, siendo mayor la caída de presión en el cabezal del orden de 5,8 psig. A nivel de laboratorio, en un reactor corto esta caída de presión va a ser menor. Davis \& Owerkerk (1979), por ejemplo, operan su reactor a 2,57 psig. Hovda (1993) en un reactor Chemithon, maneja una presión de entrada de 3 psig. De manera general, la presión de entrada va a depender del flujo de aire necesario para diluir el $\mathrm{SO}_{3}$, y del instrumento disponible para impulsar la mezcla $\mathrm{SO}_{3} / \mathrm{N}_{2}$.

Configuración de flujo en la chaqueta del reactor, los reactores industriales tienen longitudes considerables ( $6 \mathrm{~m}$ o más), por esto tienen al menos dos zonas de refrigeración con dos chaquetas independientes a temperaturas diferentes: Una en la parte superior del reactor, a baja temperatura, y otra en la parte inferior, a alta temperatura. De acuerdo con lo que se pueda presentar en la realidad experimental, se recomienda manejar en la chaqueta en la parte superior del reactor, donde las necesidades de refrigeración son altas, un flujo en contracorriente para permitir una remoción de calor mucho más eficiente, dado que es en esta parte donde se requiere un mayor control de la temperatura para evitar la pirolisis del aceite. Para la chaqueta inferior, se usa un flujo en paralelo para evitar que la temperatura en el reactor baje de un valor determinado que permita la cristalización de la materia prima o del producto sulfonado. En la parte baja, donde la viscosidad de los productos es alta, se recomienda manejar bajos flujos de agua en un intervalo de temperatura de $3^{\circ} \mathrm{C}$, al contrario de la parte alta, donde se deben manejar altos flujos y un intervalo de 1 a $2^{\circ} \mathrm{C}$.

Espesor de película, el espesor de la película se controla por medio del diseño del cabezal, del flujo del líquido y la velocidad de los gases fluyendo a través del reactor. Para flujos de metil éster bajos, el espesor de la película se mantendrá bajo. A mayor espesor 
de película, mayor resistencia a la transferencia de calor y, por tanto, mayor temperatura interfacial. Películas más delgadas proveen áreas más altas y retenciones de líquido mucho más bajas, por esto se recomiendan espesores menores a $1 \mathrm{~mm}$.

Temperatura de los reactantes, para la sulfonación de ésteres metílicos derivados del aceite de palma, la temperatura de alimentación no debe ser inferior a $40^{\circ} \mathrm{C}$, ya que por debajo de esta temperatura el éster metílico con alto contenido de metil palmitato y metil estearato entra dentro del intervalo de temperaturas de solidificación. Se recomienda temperaturas de alimentación altas $\left(60\right.$ a $\left.62^{\circ} \mathrm{C}\right)$ para que la temperatura óptima de reacción se alcance rápidamente, de tal manera que se aproveche en forma óptima la longitud del reactor. Mantener los insumos cerca de la temperatura de reacción puede hacer muy exigentes los requerimientos de calentamiento en el tanque de almacenamiento y los espesores de aislante. Por otra parte, la temperatura de entrada no debe ser demasiado alta con el fin de disminuir el riesgo de "run away" en el reactor de película.

Temperatura en la chaqueta del reactor, el agua es el refrigerante más usado por su facilidad de manejo, su bajo precio y el conocimiento que se tiene de sus propiedades térmicas. Siguiendo las indicaciones de la sección 1.3.3 para configuraciones de dos chaquetas, los valores de temperatura del agua en la chaqueta recomendados son de $50^{\circ} \mathrm{C}$ para la chaqueta superior y de $60^{\circ} \mathrm{C}$ para la chaqueta inferior. Para efectos del diseño del reactor, se considera que un reactor con 2 chaquetas brinda mucha más flexibilidad para la operación y permite un mejor manejo del calor de reacción, ya que el comportamiento térmico del reactor es distinto en la parte superior que en la parte inferior. La gran mayoría del calor de reacción se libera en los primeros $30 \mathrm{~cm}$ desde el tope del reactor de película, razón por la cual los requerimientos de refrigeración son mucho mayores en esta primera parte del reactor.

Régimen de flujo, para la sulfonación de dodecilbenceno, a nivel industrial, se manejan velocidades entre 16 y $25 \mathrm{~m} / \mathrm{s}$ en la fase gaseosa y de $2 \mathrm{~m} / \mathrm{s}$ en la fase líquida. Estas velocidades son recomendables para lograr la formación de la película, mejorar el contacto entre materias primas y asegurar flujo pistón en reactores que cuentan con un diámetro de 1 pulgada y longitudes de $6 \mathrm{~m}$ y que manejan viscosidades típicas de sulfonación que varían de 6 a 600 cP para la fase líquida con espesores de película promedios de $0.5 \mathrm{~mm}$. En reactores de laboratorio, Tano (US Patent $6,657,071$ ) por ejemplo, usa un reactor de $1 / 2$ pulgada y maneja velocidades de líquido de $0,7 \mathrm{~m} / \mathrm{s}$. y de gas de $30 \mathrm{~m} / \mathrm{s}$. Estos valores de velocidad son, no obstante, bastante altos. Un reactor de película debe manejar velocidades altas lo cual se logra o con flujos altos o con diámetros pequeños. Es preferible la segunda opción aunque el incremento del flujo de nitrógeno tiene varias ventajas para el reactor. En primer lugar, disminuye la temperatura del pico exotérmico del líquido así como la temperatura de los gases. Además de esto, de acuerdo con Davies (1972), la velocidad local de reacción se reduce debido a la disminución de la concentración del $\mathrm{SO} 3$ en la corriente gaseosa permitiendo un mejor control en la liberación de calor. Esto, porque la disminución en la fuerza impulsora para la transferencia de masa es más grande que el incremento en el coeficiente de transferencia de masa asociado con un número de Reynolds más alto. Talens et al. (1996) da importancia a la fluidodinámica del reactor indicando que flujos mayores de inerte generan esfuerzos cortantes 
más grandes en la interfase y facilitan la transferencia de masa y por lo tanto contribuye al contacto entre las especies, generando así mayores conversiones, aunque la velocidad local de reacción disminuya. El diámetro más pequeño del tubo permite también un control en la temperatura de interfase entre la corriente líquida continua y la co-corriente gaseosa al evitar las gotas de líquido que pueden ser arrastradas hacia la fase gaseosa. Los reactores de película fina descendente son apropiados para las reacciones de sulfonación, en las cuales la materia prima desciende uniformemente por las paredes del reactor y el agente sulfonante en fase gaseosa por el centro del tubo, el tipo de flujo ha de ser en paralelo, en la primera parte del reactor la reacción es muy rápida y presenta un gran incremento de la temperatura, por esta razón este control ha de realizarse con las variables de entrada al reactor.

Diseño de aparatos para procesos de sulfonación, el proceso de sulfonación lleva involucrado una reacción de dos fases, un reactante líquido correspondiente a los ésteres metílicos y el otro en fase gaseosa del agente sulfonante $\left(\mathrm{SO}_{3} / \mathrm{N}_{2}\right)$. Parte importante en el diseño corresponde a la ubicación de la planta y ha de tenerse en cuenta factores como contaminación por emisiones, disponibilidad de servicios, efectos del tránsito de personal y finalmente seguridad industrial. En la tabla 2 se listan las diferentes unidades que hacen parte de la planta de sulfonación de ésteres metílicos las cuales pueden ser observadas en el diagrama mostrado en la figura 15 donde se presenta un esquema de los equipos requeridos en el montaje de la planta. Como primer paso en el diseño se revisaron algunas condiciones de operación de trabajos reportados para la sulfonación de distintas materias primas. Varios autores presentan algunos datos que servirán de referencia para el diseño previo de la planta de sulfonación, y en particular para el cálculo de algunos de los parámetros necesarios para el diseño del reactor de película como se aprecia en la tabla 3.

Tabla 2. Equipos que conforman una planta de sulfonación

\begin{tabular}{|c|l|c|l|}
\hline 1 & Burbujeador y destilación del óleum & 13 & Tanque de almacenamiento del nitrógeno \\
\hline 2 & Tanque pulmón para el almacenamiento de $\mathrm{SO}_{3}$ & 14 & Visor de vidrio \\
\hline 3 & Deshumidificador (Demister) retenedor de gotas & 15 & Válvulas de dosificación y rotámetros \\
\hline 4 & $\begin{array}{l}\text { Intercambiador de calor de doble tubo para el gas } \\
\text { sulfonante }\end{array}$ & 16 & $\begin{array}{l}\text { Tanque de almacenamiento de } \mathrm{H}_{2} \mathrm{SO}_{4} \text { para la } \\
\text { absorción de las emisiones gaseosas de } \mathrm{SO}_{3}\end{array}$ \\
\hline 5 & Cabezal de distribución del líquido orgánico & 17 & $\begin{array}{l}\text { Tanque burbujeador de } \mathrm{SO}_{3} \text { y de recirculación } \\
\text { de NaOH para el tratamiento de emisiones }\end{array}$ \\
\hline 6 & $\begin{array}{l}\text { Reactor de película descendente FFR } \\
\text { Sulfonador) }\end{array}$ & 18 & Baños termostatados \\
\hline 7 & Salida del producto sulfonado & 19 & Columna empacada de absorción \\
\hline 8 & Baños termostatados & 20 & Manómetro \\
\hline 9 & Reactor de estabilización (Digestor) & 21 & Salida de gases \\
\hline 10 & Bombas peristálticas dosificadoras & 22 & Agitador magnético \\
\hline 11 & Depósito termostatado de líquido orgánico & 23 & Balanza digital \\
\hline 12 & $\begin{array}{l}\text { Intercambiador de calor de doble tubo para el } \\
\text { aceite }\end{array}$ & 24 & Ventilador de extracción de gases \\
\hline
\end{tabular}


Revista Publicaciones e Investigación • ISNN: 1900-6608 • Volumen 4 • Agosto de 2010

\begin{tabular}{|c|c|c|c|c|c|c|c|c|c|}
\hline | & & 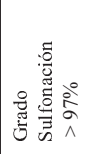 & 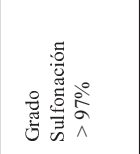 & 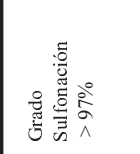 & 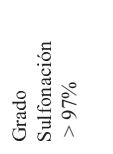 & 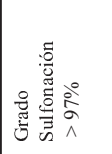 & 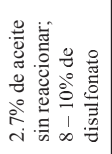 & & 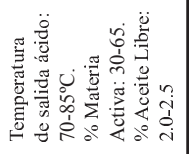 \\
\hline $\begin{array}{l}1 \\
0^{2} \\
\delta^{2}\end{array}$ & & $\bar{g}$ & $\overline{\ddot{g}}$ & $\ddot{3}$ & $\overline{\ddot{3}}$ & $\overline{\ddot{g}}$ & $\stackrel{\bar{\Xi}}{=}$ & 守 & 客 \\
\hline$\frac{1}{2}$ & & 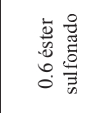 & & if & \& & $\stackrel{\triangleright}{\circ}$ & ฌ్ & $\overrightarrow{0}$ & 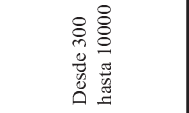 \\
\hline 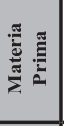 & 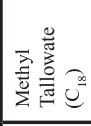 & 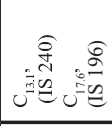 & 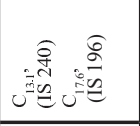 & 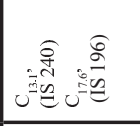 & 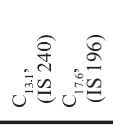 & 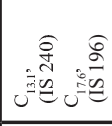 & 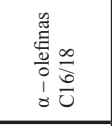 & & \\
\hline 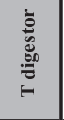 & $\begin{array}{l}0 \\
i \\
0 \\
1 \\
c \\
c\end{array}$ & $\begin{array}{l}0 \\
\stackrel{8}{0} \\
\vdots \\
\vdots \\
\infty\end{array}$ & & $\begin{array}{l}0 \\
\vdots \\
\vdots \\
\vdots \\
\infty\end{array}$ & & $\begin{array}{l}0 \\
\vdots \\
\vdots \\
0 \\
\infty\end{array}$ & & 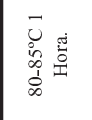 & 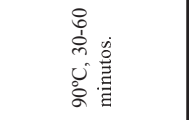 \\
\hline 离 & $\begin{array}{l}0 \\
0 \\
0 \\
0 \\
1 \\
\infty\end{array}$ & $\begin{array}{l}0 \\
\vdots \\
1 \\
2 \\
2\end{array}$ & ¿ & $\begin{array}{l}0 \\
\text { ò } \\
\text { iे } \\
2\end{array}$ & 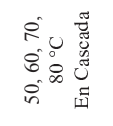 & $\begin{array}{l}0 \\
\vdots \\
\text { ò } \\
\vdots\end{array}$ & ๕. & & $\begin{array}{l}0 \\
0 \\
0 \\
\infty \\
1 \\
1\end{array}$ \\
\hline 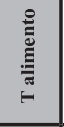 & 总 & & $\begin{array}{l}0 \\
\vdots \\
\vdots \\
1 \\
2\end{array}$ & & & & $\begin{array}{l}0 \\
0 \\
0 \\
1 \\
\text { in }\end{array}$ & 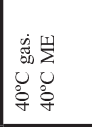 & \\
\hline ד్ & 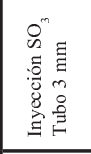 & 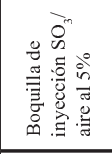 & 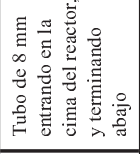 & & & & 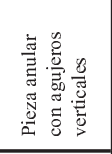 & 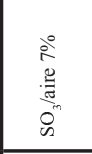 & 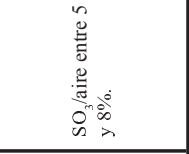 \\
\hline$\Xi$ & \pm & - & ?ै & & & & $\sim$ & & \\
\hline 急 & $\stackrel{\bar{\Xi}}{\underline{\Xi}}$ & 慁 & 言 & $\stackrel{\Xi}{\sim}$ & 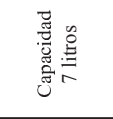 & $\stackrel{\Xi}{\Xi}$ & $\begin{array}{l}\Xi \\
\vdots \\
0 \\
1 \\
r\end{array}$ & & \\
\hline 焉 & 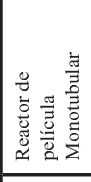 & 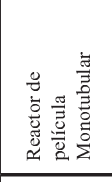 & 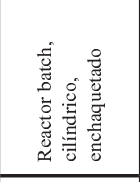 & 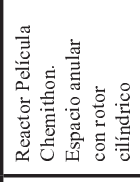 & 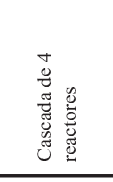 & 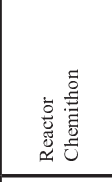 & 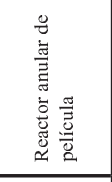 & 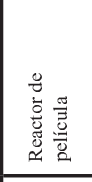 & 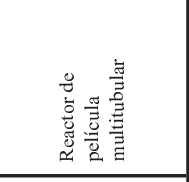 \\
\hline 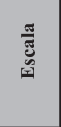 & 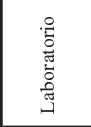 & 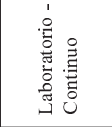 & 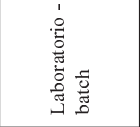 & 高 & $\frac{\circ}{\frac{\circ}{0}}$ & 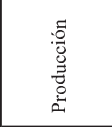 & 善 & $\frac{\circ}{\frac{\circ}{2}}$ & 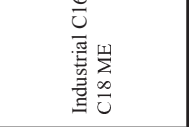 \\
\hline 彦 & 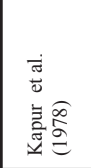 & & & 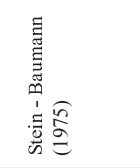 & & & 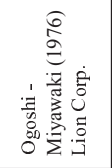 & 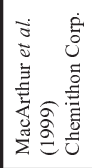 & 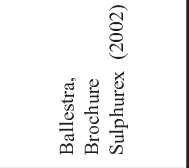 \\
\hline
\end{tabular}




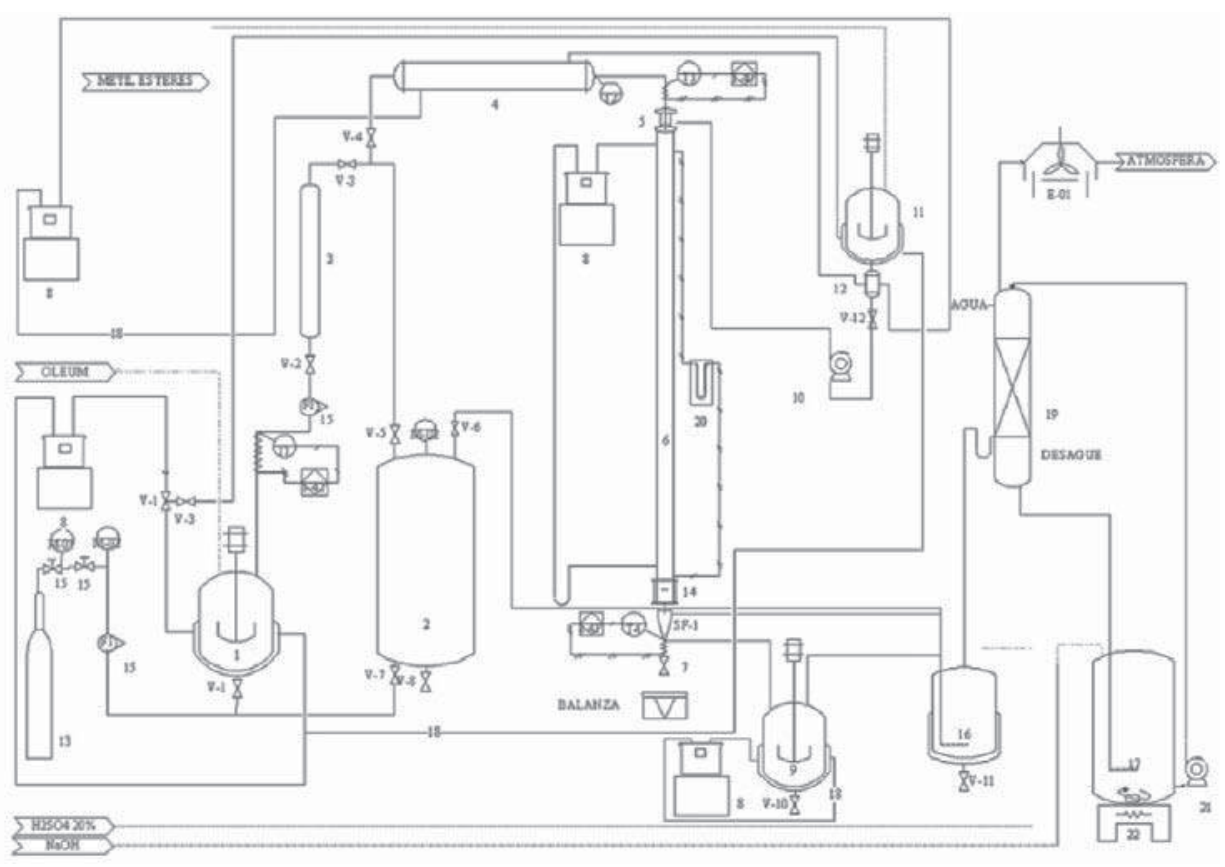

Figura 15. Esquema de la planta de sulfonación de ésteres metílicos (Torres, 2009)

En un proceso de sulfonación con $\mathrm{SO}_{3}$, previamente a la etapa de reacción es necesario implementar un sistema de tratamiento del aire utilizado para diluir el agente sulfonante, así como el propio sistema de generación de $\mathrm{SO}_{3}$. El aire que acompaña al $\mathrm{SO}_{3}$ debe ser seco para evitar que con la humedad se formen gotas de ácido sulfúrico que, además de alterar la calidad del producto que genere calor incrementando el riesgo de pirolisis del líquido orgánico. El ácido sulfúrico tiene una fuerte afinidad por el agua y forma una serie de hidratos (tales como $\mathrm{H}_{2} \mathrm{SO}_{4} \cdot \mathrm{H}_{2} \mathrm{O}, \mathrm{H}_{2} \mathrm{SO}_{4} \cdot 2 \mathrm{H}_{2} \mathrm{O}$ y $\mathrm{H}_{2} \mathrm{SO}_{4} \cdot 4 \mathrm{H}_{2} \mathrm{O}$ ). Cuando los requerimientos de humedad en el aire no son tan extremos como los mencionados, se realiza una absorción con ácido sulfúrico como agente secante. Si se requiere aire super seco, es necesario recurrir a sistemas criogénicos que permitan llegar a temperaturas que permitan la condensación de la humedad.

\section{CONCLUSIONES}

En todas estas propuestas son evidentes las partes características de un sistema de reacción para un proceso de sulfonación:

1. Almacenamiento de materias primas: Trabajan con $\mathrm{SO}_{3}$ líquido estabilizado con sulfato de dimetilo, y con alquilbencenos lineales. Ambos se almacenan en tanques confinados en cámaras termostatadas. La temperatura en la cámara de trióxido de azufre es de $35^{\circ} \mathrm{C}$. La dosificación de LAB al reactor se realiza por medio de una bomba de velocidad variable. 
2. Sistema de generación de agente de sulfonación. Es recurrente el uso de $\mathrm{SO}_{3}$ líquido estabilizado que se vaporiza, encamisado con vapor de agua. El evaporador es alimentado con una bomba jeringa con inyectores de $100 \mathrm{~mL}$ con émbolo y cabezal de teflón. Una pequeña corriente de gas inerte se usa para arrastrar el $\mathrm{SO}_{3}$ del evaporador. La mezcla entre $\mathrm{SO}_{3}$ y gas inerte se realiza en una cámara separada empacada con anillos Rasching de $5 \mathrm{~mm}$.

3. Reactor de película descendente. La reacción se hace en un reactor tubular de película como se decribe en el primer capítulo. La relación entre líquido orgánico y el $\mathrm{SO}_{3}$ se hace ajustando los flujos de cada uno antes de ingresar al reactor. Cuando se está sulfonando $\mathrm{LAB}$, no se requiere reactor de digestión, sino un reactor de neutralización. La temperatura se mide en la chaqueta, y la presión se mide en la parte superior e inferior del reactor.

4. Del sistema de tratamiento de gases no se habla mucho, pero parece ser sencillo, integrado por un burbujeador y una columna empacada. Y aunque no se mencionan es indispensable la utilización de extractores en las cercanías de la planta de sulfonación.

En suma, el sistema de reacción tendrá que tener la siguiente estructura: generación del agente sulfonante por vaporización del $\mathrm{SO}_{3}$ líquido estabilizado o destilación del oleum. La primera opción es utilizada porque permite el mejor control de la relación agente de sulfonación - fase orgánica Existen procesos a nivel industrial que usan vaporizadores trabajando entre 150 y $200^{\circ} \mathrm{C}$, para liberar el $\mathrm{SO}_{3}$ del óleum. Industrialmente, este procedimiento no es muy rentable, debido a los residuos de $\mathrm{H}_{2} \mathrm{SO}_{4}$ diluido que se generan por esta operación. Los costos de disposición del $\mathrm{H}_{2} \mathrm{SO}_{4}$ son altos y para una sulfonación a nivel industrial, se necesitaría de un óleum de por lo menos $65 \%$.

La sulfonación es un proceso que requiere una inversión de capital inicialmente exigente por cuanto es necesaria una instrumentación y un equipo especialmente diseñado para el proceso, que garantice un buen control de todos los factores mencionados. La sulfonación con $\mathrm{SO}_{3}$ reactores de película descendente, gracias a la forma de contacto entre las fases líquida y gaseosa permitieron el desarrollo de procesos de sulfonación en continuo con una sola unidad de reacción, como el que se examinará en la próxima sección. Con este aparato se tiene un mejor control de la velocidad de reacción, mayores conversiones y, en consecuencia, productos menos coloreados.

\section{REFERENCIAS BIBLIOGRÁFICAS}

ACMITE MARKET INTELLIGENCE. 2010. Market report. World Surfactant Market. (Market report Ratingen, Germany: Acmite Market Intelligence, Retrieved from http:// www.acmite.com/brochure/Brochure-Surfactant-MarketReport.pdf.

ACUÑA M. A. 2008. Es el momento de dar el salto. Palmas, 29(2): 107-118. 
Revista Especializada en Ingeniería de Procesos en Alimentos y Biomateriales. Unad.

AHMAD S., ISMAIL Z., RAFIEI A., ZAINUDIN Z., CHENG H. A. 1999. Solution properties of $\alpha$-sulfonated methyl esters and soap mixtures. Proceedings of the 1999 PORIM International Palm Oil Congress: Emerging technologies and Opportunities in the Next Millennium, 134-160.

AHMAD S., ISMAIL Z., RAFIEI A., ZAINUDIN Z., CHENG H. A. 1999. Sulphonated methyl esters from palm stearins. Proceedings of the 1999 PORIM International Palm Oil Congress: Emerging technologies and Opportunities in the Next Millennium, 275-293.

AHMAD S., ISMAIL Z., SAMSI J. 1998. Palm based sulphonated methyl esters and soap. J. Oil Palm Res., 10(1): 15-34.

AHMAD S., SIWAYANAN P., ABD MURAD Z., ABD AZIZ H., SENG SOI H. 2007. Beyond Biodiesel. Methyl Esters as the Route for the production of Surfactants Feedstock. INFORM, 18: 216-220.

AHMED Z. T., BENMAZA K., CHITOUR C. E. 2000. Sulfonation of linear alkyl benzene $(\mathrm{LAB})$ in a corrugated wall falling film reactor. Chem. Eng. J., 76: 99-102.

AKANKSHA K., PANT K., SRIVASTAVA V. K. 2007. Modeling of sulphonation of tridecylbenzene in a falling film reactor. Math. Comp. Model., 46: 1332-1344.

BACK D. D., MCCREADY M. J. 1988. Theoretical study of interfacial transport in gasliquid flours. AIChE J., 34(11): 1789-1802.

BAILEY A. E. 1996. Bailey's industrial oil and fatty products. Palm Oil. Editado por Hui, Y. H. John Whiley and Sons, Inc. 5th Edition, Vol. 2.

BAKKER B. B., CERFONTAIN H. 1989. Sulfonation of alkenes with sulfur trioxide: Reversible stereospecific $\beta$-sultone formation. Tetrahedron Letters, 30(40): 5451-5454.

BALLESTRA SPA. 2002. Ballestra Brochure. Via G. Fantloni 21/17, 20138 Milano, Italy.

BARISON Y. 2000. Future developments in the Malaysian oil palm industry: Advances in Oil Palm Research. Malaysian Palm Oil Board, 2(36): 1494-1507.

BHATTACHARYA A., GHOLAP R. V., CHAUDHARI R. V. 1988. Gas absorption with exothermic bimolecular reaction in a thin liquid film: Fast reaction. Can. J. Chem. Eng., 66: 599-604.

BATTAGLINI G., LARSON-ZOBUS J., BAKER T. G. 1986. Analytical methods for alpha sulfo methyl tallowate. JAOCS, 63(8): 1073-1077. 
BEENACKERS A., VAN SWAAIJ W. 1978. Selectivity of benzene sulphonation in three gas liquid reactors with different mass transfer characteristics. Chem. Eng. J., 15: 25-38.

BEHLER A., BIERMANN M., HILL K., RASHT H. C., SAINT V. M. E., UPHUES G. 2001. Reactions and synthesis in surfactants systems. Chapter 1: Industrial surfactant synthesis (Surfactants Science Series; 100). Edited by John Texter. Marcel Dekker, Inc. New York.

BENJUMEA P., AGUDELO J., AGUDELO A. 2008. Basic properties of palm oil biodieseldiesel blends. Fuel, 87: 2069-2075.

BENTWICH M. 1973.Combined heat and mass transfer for two reacting species with temperature dependent reaction constant. Chem. Eng. Sci., 53(7): 1465-1478.

BERNAL F. 2001. El cultivo de la palma de aceite y su beneficio. Federación Nacional de Cultivadores de Palma de Aceite - Fedepalma.

BHATTACHARYA A., GHOLAP R. V., CHAUDHARI R. V. 1988. Gas absorption with exothermic bimolecular reaction in a thin liquid film: Fast reaction. Can. J, Chem. Eng., 66: 599-604.

BOURNE J. R., VON STOCKAR U., COGGAN G. C. 1974. Gas absorption with heat effects. Ind. Eng. Chem. Process Des. Dev., 13: 124-132.

BRAD S. W., MACARTHUR B. 1996. Methyl ester sulfonate products. Chemithon. PORIM Techno. Palm Oil Res. Inst. No. 18.

BROSTRÖM A. 1974. Optimisation of a reactor of votator type for sulphonation of dodecylbencene. Trans. Instn. Chem. Engrs., 52: 384-386.

BROSTRÖM A. 1975. A mathematical model for simulating the sulphonation of dodecylbenzene with gaseous sulphur trioxide in an industrial reactor of votator type. Chem. Eng. Res. Des., 53: 29-33.

CASTAÑEDA R. W. J., RIVAS C. A. I. 2004. Dimensionamiento de un sistema para la sulfonación de ésteres metílicos del aceite de palma a nivel de laboratorio. Trabajo de grado, Departamento de Ingeniería Química, Universidad Nacional de Colombia.

CHOO Y. M., CHEAH K.Y, MA A. N., HALIM A., ONG A. S. H. 1990. Conversion of crude palm kernel oil into its methyl esters on a pilot plant scale. Proceedings of the 1990 AOCS: World Conference on Oleochemicals into the 21st Century, Edited by Thomas H. Apple white. Kuala Lumpur, Malaysia, 292-295. 
Revista Especializada en Ingeniería de Procesos en Alimentos y Biomateriales. Unad.

CLIPPINGER E. 1964. Reactions of alpha-olefins. I\&EC Prod. Res. Dev., 3(1): 3-6.

COHEN L., SOTO F., MELGAREJO A., ROBERTS D. W. 2008. Performance of $\Phi$-sulfo fatty methyl ester sulfonate versus linear alkylbenzene sulfonate, secondary alkane sulfonate and $\alpha$-sulfo fatty methyl ester sulfonate. J. Surf. Det., 11:181-186.

COHEN L., TRUJILLO F. 1998. Synthesis, characterization and surface properties of sulfoxylated methyl esters. J. Surf. Det., 1: 335-341.

DABIR B., RIAZI M. R., DAVOUDIRAD HR. 1996. Modelling of falling film reactors. Chem. Eng. Sci., 51: 2553-2558.

DAVIES J. T. 1972. Turbulence Phenomena, Academic: New York.

DAVIS E. J., VAN OUWERKERK M., VENKATESH S. 1979. An analysis of falling film gas-liquid reactor. Chem. Eng. Sci., 34: 539-550.

DE GROOT W. H. 1991. Sulphonation Technology in the Detergent Industry. Kluwer Academic Publisher. Netherlands.

DERRIG M. J., Gulf Oil Company Sales Department Brochure. March 3, 1967.

DÍAZ L. 2009. Análisis hidrodinámico de un reactor de película descendente para la sulfonación de aceite de palma derivado del aceite de palma. Tesis MSc., Universidad Nacional de Colombia, Bogotá, Colombia.

DROZD J. C. 1990. Use of sulfonated methyl esters in household cleaning products. Proceedings of the 1990 AOCS: World Conference on Oleochemicals into the $21^{\text {st }}$ Century, Edited by Thomas H. Apple white. Kuala Lumpur, Malaysia, 256-268.

DUKLER A. E., BERGELIN O. P. 1952. Characteristic of flow in falling liquid films. Chem. Eng. Prog., 48(11): 557-563.

EDWARDS B. E. 1976. The mechanism of sulphonation and sulphation. Surfactant Science Series, Anionic Surfactants Part 1. Vol. 22, Wiley Interscience, New York.

FABRY B., GIESSEN B. 1990. $\alpha$-Estersulfonate auf basis unterschiedlicher alkohole. Tenside Surf. Det., 27(4): 243-248.

FARRIS R. D. 1979. Methyl Esters in the Fatty Acid Industry, JAOCS, 56: 770A-773A.

FOSTER N. C. 2004. Soaps, detergents, oleochemicals, and personal care products. Edited by Luis Spitz. AOCS Press. Champaign, Illinois. 
FOSTER N. C. 1997. Manufacture of methyl esters sulfonates and other derivates. In presentation outlines of the Soaps, Detergents and Oleochemicals Conference and Exhibit, AOCS Publication, Chapter 9. The Chemithon Corporation, 261-287.

GILBERT E. 1965. Sulfonation and related reactions. Interscience Ed.

GILBERT E., Jones E. 1951. Sulfonation and sulfatation. Ind. Eng. Chem., 43(9): 20022052.

GILBERT E., VELDHUIS B. 1955. Sulfonation with sulfur trioxide. Ind. Eng. Chem. Prod. Dev., 47(11): 2300-2303.

GILBERT E., VELDHUIS B., CARLSON E. J., GIOLITO S. L. 1953. Sulfonation and sulfation with sulfur trioxide. Ind. Eng. Chem., 45(9): 2065-2072.

GUILLILAND E. R., SHERWOOD T. K. 1934. Diffusion of vapors into air streams. Ind. Eng. Chem., 26: 516-523.

GUTIÉRREZ J., MANS C., COSTA J. 1988. Improved mathematical model for a falling sulfonation reactor. Ind. Eng. Chem. Res., 27: 1710-1707.

HENSTOCK W., HANRATTY T. 1976. The interfacial drag and height of the wall layer in annular flows. AIChE J., 22(6): 990-1000.

HOLK N. P., VILLADSEN J. 1983. Absorption with exothermic reaction in a falling film column. Chem. Eng. Sci., 38(9): 1439-1454.

HOVDA K. 1993. Methyl ester sulphonation: process optimization. Proceedings of the 1993 PORIM International Palm Oil Congress: Update and vision chemistry and technology, 263-272.

HOWARD R. F. 1977. Chemical reactor design for process plants. John Wiley \& Sons, Inc.

HOYT J. L., SONES E. L., SOOTER A. J. 1979. Separation and quantitative determination of active ingredients in detergent formulations. JAOCS, 56: 701-703.

HULBERT R. C., KNOTT R. F., CHENEY H. A. 1967. Apparatus for small scale sulfonation with SO3. Soap Chem. Spec., 42(6): 88-134.

INAGAKI T. 1990. Development of $\alpha$-sulfo fatty acid esters. Proceedings of the 1990 AOCS: World Conference on Oleochemicals into the 21 st Century, Edited by Thomas H. Applewhite. Kuala Lumpur, Malaysia, 269-271. 
Revista Especializada en Ingeniería de Procesos en Alimentos y Biomateriales. Unad.

INGEGARD J., MARTIN S. 2001. Surfactans based on fatty acids and other natural hydrophobes. Coll. Int. Sci., 6: 178-188.

IRAZOQUI H. A., ISLA M. A. 1996. La Oleoquímica. Usos alternativos de los aceites vegetales, productos y aplicaciones de mayor valor comercial a partir de los ésteres y de la glicerina. Aceites y Grasas, 2(23): 171-188.

IRAZOQUI H. A., ISLA M. A. 1996. Oportunidades de investigación y desarrollo en el área de la Oleoquímica a partir de aceites vegetales, producción de moléculas precursoras. Aceites y Grasas, 2(23): 192-198.

ISMAIL Z., AHMAD S., Greentech Engineering SDN. 1997. Palm-based anionic surfactants. PORIM Information Series No. 48.

ISMAIL Z., AOKI K., YONEYAMA Y., MUIKAIYAMA T., SATSUKI T., AHMAD S. 1999. Solution properties of $\alpha$-sulfonated methyl esters and soap mixtures. Proceedings of 1999 PORIM International Palm Oil Congress: Emerging Technologies and Opportunities in the next Millennium. Kuala Lumpur, Malaysia, 134-160.

JOHNSON G. R., CRYNES B. L. 1974. Modelling of a thin-film sulfur trioxide sulfonation reactor. Ind. Eng. Chem. Process Des. Dev., 13: 6-14.

KAPUR B. L., SOLOMON J. M., BLUESTEIN B. R. 1978. Summary of the technology for the manufacture of higher alpha-sulfo fatty acid esters. JAOCS, 55: 549-557.

KARIMI G., KAWAJI M. 1998. An experimental study of freely falling films in a vertical tube. Chem. Eng. Sci., 53(20): 3501-3512.

KERN D. Q. 1992. Procesos de transferencia de calor. Compañía Editorial Continental, S. A. México.

KIRCHER J. E., MILLER E. L, GEISER P. E. 1954. Sulfonation of detergent alkylates. Ind. Eng. Chem., 46(9): 1925-1930.

KNAGGS E. 2004. Comment on "Optimization of the linear alkyl benzene sulfonation process for surfactant manufacture" and "Sulfonation technology for anionic surfactant manufacture": Falling Film SO3 Sulfonation - Laminar or Turbulent Flow Controversy. Org. Process Res. Dev., 8(6): 1079-1080.

KNAGGS E., NEPRAS M. J. 1997. Sulfonation and sulfation. Kirk-Othmer Encyclopedia of Chemical Technology, 4th edition; John Wiley \& Sons. New York, 23: 146-193.

KOCAMUSTAFAOGULLARI G., CHEN I. Y. 1988. Falling film heat transfer analysis on a bank of horizontal tube evaporator. AIChE J., 34: 1539-1549. 
KOHASHI H. 1990. Application of fatty acid esters for lubricating oil. AOCS. Proceedings of the 1990 AOCS: World Conference on Oleochemicals into the 21st Century, Edited by Thomas H. Applewhite. Kuala Lumpur, Malaysia, 243-250.

KUO-YANN L. 2006. Liquid detergents. CRC Press, Taylor \& Francis Group. Second Edition. Surfactant Science Series, Vol. 129, New Jersey, USA.

LAMOURELLE A., SANDALL O. 1972. Gas absorption into a turbulent liquid. Chem. Eng. Sci., 27: 1035-1043.

LANTERI A. 1978. Sulfonation and sulfation technology. JAOCS, 55: 128-133.

LEDBETTER L., MACARTHUR B. W., BROOKS B. 2000. Novel Drying Process Applied to Anionic surfactants. The Chemithon Corporation, USA.

LEONG W. L., MOHD A. G. 1989. Production of value added products by processing palm fatty acid distillates. The Planter, 65: 764.

LEWANDOWSKI H, SCHWUGER M. J. 2003. Novel surfactants, preparations, applications and biodegradability. Chapter 13: $\alpha$-sulfomonocarboxylic esters. Edited by K. Holmberg. Second edition. Marcel Dekker, Inc. New York.

LIM W. H., AHMAD S. 2001. Surface tension, foaming and detergency properties of mixed alpha-sulphonated methyl esters derived from palm stearin with some commercial surfactants. J. Oil Palm Res., 13(1): 75-83.

LIM W. H., AHMAD S., ISMAIL Z. 2000. Physic-chemical properties of binary systems of alpha-sulphonated methyl ester derived from palm stearin and anionic surfactants. J. Oil Palm Res., 12(2): 20-28.

Lim W. H., Ho S. G. 1999. Properties of mixed alpha sulfonated methyl ester derived from palm stearin and polyoxyethylene sorbitan monoester in diluted solution. Proceedings of 1999 PORIM International Palm Oil Congress: Emerging Technologies and Opportunities in the Next Millennium. Kuala Lumpur, Malaysia, 161-176.

LINFIELD W. M. 1970. Cationic surfactants, Chap. 2. (E. Jurgermann, Ed.), Marcel Dekker, Inc., New York.

MACARTHUR B. W., BROOKS B., SHEATS W. B., FOSTER N. C. 1999. Meeting the challenge of methyl ester sulfonation. Proceedings of the 1999 PORIM International Palm Oil Congress: Emerging technologies and Opportunities in the Next Millennium, 229-250. 
Revista Especializada en Ingeniería de Procesos en Alimentos y Biomateriales. Unad.

MANN R., KNISH P., ALLAN J. C. 1982. Exothermic gas absorption with chemical complex reaction. J. Weil \& K. C. Gerogakis. ACS Symposium Series 196, 441-456.

MANN R., MOYES H. 1977. Exothermic gas absorption with chemical reaction. AIChE J., 23(1): 17-23.

MAURER E. W., WEIL J. K., LINFIELD W. M. 1977. The biodegradation of esters of $\alpha$-sulfo fatty acids. JAOCS, 54: 582-584.

MAZZONI G. SPA. 1986. $\mathrm{SO}_{3}$ sulfonation/sulfation processing technology brochure. P. O. Box 421, 21052 - Busto Arsizio, Italy.

MCCREADY M. J., HANRATTY T. J. 1984. Gas transfer at water surfaces. Vol. 283. D. Reidel Dordrecht, Holland: Publishing Company.

MERCHUK J. C., FARINA I. H. 1976. Simultaneous diffusion and chemical reaction in twophase systems. Chem Eng. Sci., Vol. 31, $645-650$.

MESA D. J. 2006. Nuevas oportunidades para un posicionamiento estratégico del aceite de palma en el mercado mundial. XV Conferencia Internacional sobre Palma de Aceite. Federación Nacional de Cultivadores de Palma de Aceite - Fedepalma.

MESA D. J. 2006. La Agroindustria de la Palma de Aceite en Colombia. Federación Nacional de Cultivadores de Palma de Aceite - Fedepalma.

MESA D. J. 2008. Biodiesel de palma: un proyecto de largo aliento convertido en realidad. Palmas, 29: 1.

MILWDSKY B. M., GABRIEL D. M. 1982. Detergents Analysis - A Handbook for costeffective quality control. Publisher by George Godwin, London.

MORETTI G. F., ADAMI I. 1998. Fatty acid methyl Esther's in the detergent industry. R\&D Department - Ballestra SpA.

MORETTI G. F., ADAMI I. 2000. Production, processing and uses of methyl esters and derivate. R\&D Department - Ballestra SpA.

MORETTI G. F., ADAMI I. 2002. SO3-Sulphonation: Evolution of process design as a function of update feedstock and surfactant quality specifications. R\&D Department Ballestra S.p.A.

MORETTI G. F., ADAMI I., NAVA F. 1994. High-Purity, Concentrated Anionic Surfactants from Improved Sulfonation and Vacuum-Neutralization Technology. R\&D Department 
- Ballestra SpA. In: Proceedings of the $3^{\text {rd }}$ World Conference on Detergents: Global Perspectives. Editor: Arno Cahn. AOCS Press. New York, 267-272.

MUDAWAR I., HOUPT R. 1993. Measurement of mass and momentum transport in wavylaminar falling liquid films. Int. J. Heat Mass Transfer, 36(17): 4151-4162.

NAGAYAMA M., OKUMA O., SAKATANI T., HASHIMOTO S., NODA S. 1975. Yukagaku, 24, 395; C.A. 83:130805 1975.

NARVÁEZ R. P. C., SÁNCHEZ C. F. J., TORRES O. J. A., PONCE DE LEÓN Q. L. F. 2004. Influencia de la temperatura y el porcentaje del catalizador en la etanólisis del aceite de palma. Revista Colombiana de Química, 23(2): 109-115.

NARVÁEZ R. P. C., SÁNCHEZ C. F. J., TORRES O. J. A., PONCE DE LEÓN Q. L. F. 2004. Producción de ésteres metílicos de ácidos grasos: variables asociadas al proceso de transformación. Ing. Investig., 55: 41-50.

NARVÁEZ R. P. C., TORRES O. J. A., SÁNCHEZ C. F. J., PONCE DE LEÓN Q. L. F. 2005. Determinación por cromatografía de gases de alquil ésteres (metilo y etilo) de ácidos grasos. Ing. Investig., 57: 58-62.

NDOUMBE S., LUSSEYRAN F., IZRAR B. 2003. Contribution to the modeling of a liquid film flowing down inside a vertical circular tube. C. R. Mécanique, 331: 173-178.

NIELSEN P. H, VILLADSEN J. 1983. Absorption with exothermic reaction in a film column. Chem. Eng. Sci., 38: 1439-1454.

NIELSEN P., VILLADSEN J. 1985. An analysis of the multiplicity pattern of models for simultaneous diffusion, chemical reaction and absorption. Chem Eng. Sci., 40: 571-587.

ONG A. S. H, KIFLI H., HASSAN H., CHOW C. S. 1990. Palm oil as oleochemicals raw materials. Proceedings of the 1990 AOCS: World Conference on Oleochemicals into the $2{ }^{\text {st }}$ Century, Edited by Thomas H. Applewhite. Kuala Lumpur, Malaysia, 82-87.

OWEN D. F. G., HEWITT G. F. 1987. An improved annular two-phase flow model. $3^{\text {rd }}$ International Conference on multiphase flow (BHRA), The Hague.

PORTALSKI S., CLEGG A. J. 1972. An experimental study of wave inception on falling liquid films. Chem. Eng. Sci., 27: 1257-1265.

PRYDE E. H. 1979. Fat and oils as chemical intermediates: present and future uses. JAOCS, 56(8) 849-854. 
Revista Especializada en Ingeniería de Procesos en Alimentos y Biomateriales. Unad.

RASE H. F. 1977. Chemical reactor design for process plants. Volume 2: Case of studies and design data. CS 107. John Wiley \& Sons Inc. New York.

RESNIK G. O., VISHWANATH P., PYNN M. A., SITNIK J. M., TODD J. J., WU, J., . . JARRELL, K. A. 2010. Use of sustainable chemistry to produce an acyl amino acid surfactant. Appl. Microbiol. Biot., Vol.85, No. 5, pp.1387-1397.

RIAZI M., FAGHRI A. 1986. Gas absorption with zero-order chemical reaction. AIChE J., 31(12): 1967-1972.

ROBERTS D. W. 1992. Qualitative correlations for the effects of chain branching on aquatic toxicity and micellisation of anionic surfactants. Jorn. Com. Esp. Deterg., 23: 81-92.

ROBERTS, D. W. 1998. Sulfonation technology for anionic surfactant manufacture. Org. Process Res. Dev., 2(3): 194-202.

ROBERTS D. W. 2001. Chapter 3: Manufacture of anionic surfactants. Oleochemical Manufacture and Applications. Editado por Frank D. Gustone and R. J. Hamilton. Sheffield Academic Press. CRC Press UK. 55-73.

ROBERTS D. W. 2003. Optimization of linear alkyl benzene sulfonation process for surfactant manufacture. Org. Process Res. Dev., 7(2): 172-184.

ROBERTS D. W. 2004. Reply to: Comment on "Optimization of the linear alkyl benzene sulfonation process for surfactant manufacture" and "Sulfonation technology for anionic surfactant manufacture": Falling Film $\mathrm{SO}_{3}$ Sulfonation - Laminar or Turbulent Flow Controversy. Org. Process Res. Dev., 8(6): 1081-1082.

ROBERTS D. W. 2007. The origin of color formation in methyl ester sulfonation. Jorn. Com. Esp. Deterg., 37: 153-159.

ROBERTS D. W., GARRET P. 1999. Esther sulphonates derived from non-methyl esters. Proceedings of the 1999 PORIM International Palm Oil Congress: Emerging technologies and Opportunities in the Next Millennium, 209-215.

ROBERTS D. W., GIUSTI L, FORCELLA A. 2008. Chemistry of methyl ester sulfonates. A supplement special: Surfactants from Biorenewable Resources. INFORM 5: 2-9.

ROBERTS D. W., JACKSON P. S., SAUL C. D., CLEMENT C. J., ALLAN A., HODGE R. A. 1995. Intermediate by-products in methyl ester sulphonation. Jorn. Com. Esp. Deterg., 26: $27-37$.

ROBERTS D. W., JACKSON P. S., SAUL C. D., CLEMETT C. J., JONES K. 1988. A kinetic and mechanistic investigation of ester sulphonation. Proceedings of $2^{\text {nd }}$ World Surfactants 
Congress. Paris. ASPA (Synidcat National des Fabricants d'Agents de Surface et Produits Auxiliares Industriels). Vol II, 38-41.

SATSUKI T. 1992. Applications of MES in Detergents. INFORM 3: 1099-1108.

SATSUKI T. 1994. Methyl ester sulfonates: A surfactantant based on natural fats. Lion Corporation, Fabric Care Research Labs. In: Proceedings of the $3^{\text {rd }}$ World Conference on Detergents: Global Perspectives. Editor: Arno Cahn. AOCS Press. New York, 135-140.

SCHAMBIL F., SCHWUGER M. J. 1990. Physico-chemical properties of alpha-sulpho fatty acid methyl esters and alpha-sulpho fatty acid di salts. Tenside, 27(6): 380-385.

SHEATS W. B., MACARTHUR B. W. 2000. Methyl ester sulfonate products. The Chemithon Corporation.

SMITH F. D., STIRTON A. J. 1967. The alpha-sulphonation of alkyl palmitates and stearates. JAOCS, 44: 405.

SCHMITT T. M. 2001. Analysis of surfactant. Second Edition. Marcel Dekker, Inc. New York.

SONNTAG N. O. V. 1979. New developments in the fatty acid industry. JAOCS, 56(11): 861A-864A.

STEIN W., BAUMANN H. 1975. $\alpha$-sulfonated fatty acids and esters: manufacturing process, properties, and applications. JAOCS, 52: 323-329.

STEIN W., WEISS H., KOCHO O., NEUHASEN P., BAUMANN H. 1970 Fette Steifen Anstrichm, 72: 956.

STIRTON A. J. 1962. $\alpha$-Sulfo fatty acids and derivates synthesis, properties and use. JAOCS, 39: 490-496.

STIRTON A. J., BISTLINE R. G., BARR E. A., NUÑEZ-PONZOA M. V. 1965. Salts of alkyl esters of $\alpha$-sulfopalmitic and $\alpha$-sulfostearic acids. JAOCS, 42: 1078-1081.

STIRTON A. J, WEIL J. K., BISTLINE R. G. 1962. Sodium salts of alkyl esters of $\alpha$-sulfo fatty acids, wetting, lime soap dispersion and related properties. JAOCS, 39: 128.

STIRTON A. J., WEIL J. K., BISTLINE R. G. 1954. Surface-active properties of salts of alpha-sulfonated acids and esters. JAOCS, 31: 13-16.

TALENS A. F. I. 1999. The modeling of falling film chemical reactor. Chem. Eng. Sci., 54 : 1871-1881. 
Revista Especializada en Ingeniería de Procesos en Alimentos y Biomateriales. Unad.

TALENS A. F. I. 2000. The effect of superficial gas velocity on wavy films and its use in enhancing the performance of falling film reactor. Chem. Eng. Technol., 23(7): 629-6332.

TALENS A. F, I., GUTIÉRREZ J. M. 1995. Estimation of the viscosity and density of lauryl alcohol/laurylsulphuric acid mixtures as a function of the temperature and laurylsulphuric and molar fraction. Chem. Eng. Res. Des., 73 A: 206-207.

TALENS A. F. I., GUTIÉRREZ J. M., MANS C. 1994. Effect of surface tension and viscosity on the efficiency in wetted wall reactors. Tenside Surf. Det., 31(1): 6-8.

TALENS A. F. I., GUTIÉRREZ J. M., MANS C. 1996a. An improved falling-Film reactor for viscous liquid. JAOCS, 73(7): 857-861.

TALENS A. F. I., PEREIRA G., CHENLO-ROMERO F., VÁZQUEZ U. 1996b. Device for research into falling film wave structures under shear stress. AIChE J., 42(11): 3293-3295.

TORRES, O. J. A. 2009. Producción de alfa-sulfo metil estearato en un reactor de película líquida descendente. Tesis doctoral, Departamento de Ingeniería Química, Universidad Nacional de Colombia, Bogotá, Colombia.

TORRES O. J. A, DÍAZ A. L. A, SÁNCHEZ C. F. J. 2009a. Reactor de película líquida descendente para la sulfonación de esteres metílicos con trióxido de azufre. Ing. Investig., 29(3): 48-53.

TORRES O. J. A., DÍAZ A. L. A., SÁNCHEZ C. F. J. 2008a. Estudio cinético de la sulfonación de metil ésteres derivados del aceite de palma (Elaeis guineensis). Corpoica Cienc. Tecnol. Agropecu., 9(2): 88-95.

TORRES, J. A., MORAlES, G. M., SUÁREZ, P. O. Y., \& SÁNCHEZ, F. J. $2009 b$. Mathematical model of a falling film reactor for methyl ester sulfonation. Chem. Prod. Process Model. Vol. 4(5), Art. 12.

TORRES O. J. A., SÁNCHEZ C. F. J. 2007. Propiedades fisicoquímicas de la mezcla líquida de estearato de metilo / ácido sulfo estearato de metilo. Publ. Investig., 2(1): 97-118.

TORRES O. J. A., SÁNCHEZ C. F. J., NARVÁEZ R. P. C., PONCE DE LEÓN Q. L. F. 2005. Obtención de estearato de metilo derivado de la estearina hidrogenada derivada de aceite de palma. Revista de Investigaciones de la UNAD, 4(2): 150-162.

TORRES O. J. A., SÁNCHEZ C. F. J., SUAREZ P. O. Y., NARVÁEZ R. P. C. 2008 b. Sulfonación de ésteres metílicos derivados del aceite de palma (Elaeis guineensis) a escala laboratorio con SO3. Corpoica Cienc. Tecnol. Agropecu. 9(2): 77-87. 
TOYODA S., OHGOSHI T., KITANO K., MIYAWAKI Y. 1982. Kagaku Sochi., 24: 101.

TSUBOCHI M., YAMASAKIN., MATSUOKAK. 1979. Determination of anionic surfactants by two-phase titration with tetrabromophenolphthalein ethyl ester as indicator. J. Am. Chem. Soc., 56(11): 921-923.

URIBE E. 2008. Reflexiones sobre el crecimiento del sector agrícola en Colombia. Palmas, 29(2): 91-102.

VAN DAM M. H., CORRIOU J. P., MIDOUX A., LAMINE S., ROIZARD C. 1999. Modeling and measurement of sulfur dioxide absorption rate in a laminar falling film reactor. Chem. Eng. Sci., 54: 5311-5318.

VILLADSEN J., NIELSEN H. P. 1986. Models for strongly exothermic absorption and reaction in falling films. Chem. Eng. Sci., 41(6): 1655-1671.

WEIL J. K., WITNAUER L. P., STIRTON A. J. 1953. Evidence for $\alpha$-sulfonation in the reaction of palmitic acid with sulfur trioxide. J. Am. Chem. Soc., 75: 2526-2527.

WHALLEY G. R. 1991. Toilet soaps from fatty methyl esters. HAPPI 4, 81-84.

WOOD B. J. 2008. Opportunities for oil palm R\&D in further meeting the challenges of the new dynamics. Palmas, 29(1): 59-79.

YAMADA K., MATSUTANI S. 1993. Separation and identification of colored substances in sulfonated fatty acid methyl ester, presented at $84^{\text {th }}$ AOCS Annual Meeting and Expo, Anaheim, California.

YAMADA K., MATSUTANI S. 1996. Analysis of the dark colored impurities in sulfonates fatty acid methyl ester. JAOCS, 73: 121-125.

YAMAGUCHI S. 1978. Determination of sodium sulfate in anionic surfactants by nonaqueous titration. JAOCS, 55: 673-674.

YIH S., LIU J. 1983. Prediction of heat transfer in turbulent falling liquid films with or whitout interfacial shear. AIChE J., 29: 903-909.

YOUNG D. C. 2001. Computacional Chemitry. Chapter 35. Wiley-Interscience. John Wiley $\&$ Sons, Inc. New York.

\section{PATENTES}

United States Patent 1,081,775 Russ, Rudolf. Method of obtaining sulfonated oils and fats. 16th December 1913. 
Revista Especializada en Ingeniería de Procesos en Alimentos y Biomateriales. Unad.

United States Patent 1,185,213 Levinstein, Ivan. Production of sulfonated palmitic products. $30^{\text {th }}$ May 1916.

United States Patent 1,321,994. Sulfonation of hydrocarbon with SO3. Downs, Charles E. 18th November 1919.

United States Patent 1,926,442 Günther, Fritz; Hetzer, Josef. Production of sulfonic acids of aliphatic and hydroaromatic carboxylic acids. 12 ${ }^{\text {th }}$ September 1933.

United States Patent 2,088,027 Law, George H; McNamee, Raymond. Alcohol sulfation process. July 271937 Union Carbide and Carbon Corporation.

United States Patent 2,195,145 Syracuse, Eugene D. Crittenden. Production of sulfonation derivatives. The Solvay Process Company. 26 March 1940.

United States Patent 2,195,186; 2,195,187 y 2,195,188, Moyer W.W. (1940).

United States Patent 2,195,187 Wendell W. Moyer; Decatur. Production of sulfonation derivatives. The Solvay Process Company. 12th September 1938.

United States Patent 2,352,698 Eaton T, James; Wyncote, Volk, Peter W. Process of preparing a sulfonated product. $14^{\text {th }}$ July 1944 .

United States Patent 2,743,288 Rueggeberg, Walter; Sauls, Thomas. Production of monosulfonated carboxylic acids and their esters. 24th June 1953.

United States Patent 2,923,728 Falk, Klaus; Wilmington; Taplin Walter Renold. Process of reacting organic compounds with sulfur trioxide. 2th February 1960 Du Pont Nemours and Company.

United States Patent 3,158,632 Blaser, Bruno; Stein Werner, Weiss Herber. $\alpha-$ sulfonation of saturated fatty acids and their derivatives. $24^{\text {th }}$ November 1964 Henkel Co.

United States Patent 3,169,142 Knaggs, et al. Method for sulfonation and sulfation of organic compounds. 9th February 1965 STEPAN.

United States Patent 3,256,303 Stein, et al. Sulfonation of fatty acids and their esters Henkel $12^{\text {th }}$ October 1966.

United States Patent 3,531, 518 Jacobsen R. L., Ohren T. H. September 29, 1970.

United States Patent 3,931,273. Method for sulfonating and sulfating compounds with sulfur trioxide and apparatus. Lanteri, A. 12th March 1978. 
United States Patent 3,997,576. Method for preparation of alpha-sulfofatty acid ester. $14^{\text {th }}$ December 1976. Oghoshi; Toshiaki (Funabashi, JA); Kusumi; Yukio (Chiba, JA Lion Fat \& Oil Co., Ltd. (Tokyo, JA).

United States Patent 4,080,372. Continuous process for bleaching acid alpha-sulfo-fatty acid esters. 21th March 1978. Stein; Werner (Dusseldorf, DT); Baumann; Horst (Leichlingen, DT); Rommerskirchen; Hans Josef (Hilden, DT). Henkel Kommanditgesellschaft auf Aktien (Henkel KGaA) (Dusseldorf-Holthausen, DT.

United States Patent 4,113,438. Sulfonating Apparatus. 12 ${ }^{\text {th }}$ September 1978 Brooks et. al. The Chemithon Corporation.

United States Patent 4,308,215 Vaughan, Ronald, Sulfonation Process. Varen Technology, $26^{\text {th }}$ December 1981.

United States Patent No. 4,671,900, Preparation of Light-colored wash active $\alpha$-Sulfonatty Acid, Schmid, K., Stein, W. and Baumann, H. 31th January 1987.

United States Patent No. 4,990,288, Control of disalt in alpha sulfofatty acid ester surfactants. Febrero 15 de 1991.

United States Patent 5,136,088. Sulfonation process for viscous sulfonic acids. Farmer et al. Agosto 12 de 1992.

United States Patent 5,382,677. Process for the production of highly concentrated pastes of alpha-sulfofatty acid alkyl éster alkali metal salts. January 17 de 1995. Colignon; Dietmar (Erkrath, DE); Dorra; Erich (Duesseldorf, DE); Panthel; Guenter (Haan, DE); Schmidt; Wolfgang (Monheim, DE); Wrede; Norbert (Duesseldorf, DE). Henkel Kommanditgesellschaft auf Aktien (Duesseldorf, DE).

United States Patent 5,391,783. Process for the production of light-colored pastes of alphasulfofatty acid alkyl éster alkali metal salts. February 21, 1995 Colignon; Dietmar (Erkrath, DE); Dorra; Erich (Duesseldorf, DE); Lepper; Herbert (Hilden, DE); Panthel; Guenter (Haan, DE); Pierron; Francois (Annecy, DE); Schmidt; Wolfgang (Monheim, DE); Wrede; Norbert (Dusseldorf, DE). Henkel Kommanditgesellschaft auf Aktien (Duesseldorf, DE).

United States Patent 5,391,786. Process for the production of light-colored washingactive alpha-sulfofatty acid lower alkyl ester salts. February 21 de 1995. Fabry; Bernd (Korschenbroich, DE); Kratzel; Ulrich (Kulmbach, DE); Schmidt; Wolfgang (Monheim, DE); Kreienfeld; Guenter (Duesseldorf, DE). Henkel Kommanditgesellschaft auf Aktien Duesseldorf, DE). 
Revista Especializada en Ingeniería de Procesos en Alimentos y Biomateriales. Unad.

United States Patent 5,429,773. Process to improve alkyl ester sulfonate surfactant compositions. July 4 de 1995 Sherry; Alan E. (Cincinnati, OH); Chapman; Benjamin E. (Cincinnati, OH); Creedon; Michael T. (Cincinnati, OH). The Procter \& Gamble Company (Cincinnati, $\mathrm{OH})$.

United States Patent 5,587,500. Sulfonation of fatty acid esters. Hovda; Keith D. (Kent, WA). The Chemithon Corporation (Seattle, WA) Diciembre 24, 1996.

United States Patent 5,723,433. Solvent removal process. March 3 de 1998. Duvall; Lanny R. (Kent, WA); Brooks; Burton (Bellevue, WA); Jessup; Walter (Seattle, WA). The Chemithon Corporation (Seattle, WA).

United States Patent No. 5,136,088. Sulfonation Process for viscous sulfonic acids, Farmer et. al. The Chemithon Corporation. Agosto 2 de 1992.

United States Patent No. 6,657,071, Tano et al., to Lion Corporation. Diciembre 2 de 2003. 